\title{
Energy Harvesting Noncoherent Cooperative Communications
}

\author{
Peng Liu, Saeed Gazor, Il-Min Kim, and Dong In Kim
}

\begin{abstract}
This paper investigates simultaneous wireless information and power transfer (SWIPT) in energy harvesting (EH) relay systems. Unlike existing SWIPT schemes requiring the channel state information (CSI) for coherent information delivery, we propose a noncoherent SWIPT framework for decodeand-forward (DF) relay systems bypassing the need for CSI and consequently saving energy in the network. The proposed SWIPT framework embraces power-splitting noncoherent DF (PS-NcDF) and time-switching noncoherent DF (TS-NcDF) in a unified form, and supports arbitrary $M$-ary noncoherent frequency-shift keying (FSK) and differential phase-shift keying (DPSK). Exact (noncoherent) maximum-likelihood detectors (MLDs) for PS-NcDF and TS-NcDF are developed in a unified form, which involves integral evaluations yet serves as the optimum performance benchmark for noncoherent SWIPT. To reduce the computational cost of the exact MLDs, we also propose closed-form approximate MLDs achieving near-optimum performance, thus serving as a practical solution for noncoherent SWIPT. Numerical results demonstrate a performance tradeoff between the first and second hops through the adjustment of time switching or power splitting parameters, whose optimal values minimizing the symbol-error rate (SER) are strictly between 0 and 1 . We demonstrate that $M$-FSK results in a significant energy saving over $M$-DPSK for $M \geq 8$; thus $M$-FSK may be more suitable for EH relay systems.
\end{abstract}

\section{Index Terms}

Energy harvesting, maximum likelihood, noncoherent SWIPT, wireless information transfer (WIT), wireless power transfer (WPT).

P. Liu is with the Department of Electrical Engineering, Stanford University, Stanford, CA, 94305, USA (e-mail: pengliu1@stanford.edu).

S. Gazor and I.-M. Kim are with the Department of Electrical and Computer Engineering, Queen's University, Kingston, Ontario, K7L 3N6, Canada (e-mail: gazor@queensu.ca; ilmin.kim@queensu.ca).

D. I. Kim is with the school of Information and Communication Engineering, Sungkyunkwan University (SKKU), Suwon, Korea (e-mail: dikim@skku.ac.kr) 


\section{INTRODUCTION}

The wireless information transfer (WIT) and wireless power transfer (WPT) technologies share the common origin of the electromagnetic waves, which is a direct consequence of the fact that radio frequency $(\mathrm{RF})$ signals carry energy as well as information at the same time. Nevertheless, prior research activities and industrial developments on WIT have been carried out independently from those on WPT, due to the fact that WIT and WPT operate with rather different receiver power sensitivities, i.e., $-60 \mathrm{dBm}$ for information receivers and $-10 \mathrm{dBm}$ for energy receivers [1], [2]. As a result, WIT and WPT have distinct receiver architectures and design objectives. In particular, WIT makes use of the active mixer for maximal information delivery rate [3], whereas WPT utilizes the passive rectenna for maximal energy transmission efficiency [4]. It is not until recently that the dual use of RF signals for simultaneous wireless information and power transfer (SWIPT) [5], [6] has been studied, giving rise to an appealing solution for energy-constrained wireless systems such as cooperative relay networks.

\section{A. Related Works}

Recent advances on SWIPT have demonstrated conflicting objectives in designing WIT for maximal information delivery rate and WPT for maximal energy transmission efficiency, as witnessed by the fundamental rate-energy tradeoff [5]. Varshney first introduced the notion of the rate-energy region for SWIPT in additive white Gaussian noise (AWGN) channels [5], which was then generalized to frequency-selective fading channels [6]. These pioneering studies, however, were based on an ideal receiver circuit which simultaneously decodes information and harvests energy from the same signal without any loss [5], [6]. It has been recognized that this ideal receiver architecture [5], [6] may be difficult to implement given the current state-of-the-art of electronic circuits. A practical receiver architecture incorporating both energy harvesting $(\mathrm{EH})$ and information decoding (ID) circuits into a single terminal was proposed in [7]. Based on this practical architecture, the wireless terminal may operate either in the time switching mode [8], in which the receiver perform EH and ID in a time-division fashion, or in the power splitting mode [9], in which the receiver splits the received signal into two streams for separate EH and ID at the same time. The corresponding rate-energy tradeoff was studied for single-input single-output (SISO) [2], [9] and multiple-input multiple-output (MIMO) channels [7]. The power allocation, beamforming design, and optimization schemes were studied to achieve various tradeoffs between 
$\mathrm{EH}$ and ID. The throughput maximization for a multi-user system was addressed in [10]. The optimum power allocation maximizing the information rate while guaranteeing that the harvested energy is above a threshold was studied for broadband systems where perfect instantaneous channel state information (CSI) was available at the transmitter [11]. Moreover, beamforming scheme maximizing the harvested energy subject to information rate constraint was studied under imperfect CSI in [12]. Similarly, beamforming design maximizing the information rate subject to EH constraint was addressed in [13]. The precoder maximizing the energy efficiency in MIMO wiretap channel subject to secrecy rate and power constraints was studied in [14]. These works [2], [5]-[14] represent typical applications of SWIPT in point-to-point (P2P) communications.

Recently, the SWIPT has also found its application in energy-constrained wireless relay networks. In EH relay systems, the source and destination typically have dedicated power supplies from battery or power grid, while the relays are powered by the RF signals radiated by the source. The throughput performance of an amplify-and-forward (AF) relay network employing EH relays was studied for both delay-limited and delay-tolerant applications in [15]. The benefits of EH relays and the differences between EH relay networks and conventional relay networks were investigated in [16]. In [17], a joint power allocation and relay selection scheme for AF relay networks with EH ability was studied. A greedy switching strategy between EH and data relaying was investigated for a dual-hop AF network, and the outage probability was analyzed in [18]. Besides AF relay systems, SWIPT has also been studied for decode-and-forward (DF) relay systems. Specifically, a DF network consisting of multiple source-destination pairs and one EH relay was studied in [19], where the outage probability was studied taking into account the spatial randomness of the source-destination pairs. For the same system, the diversity performance and power allocation were studied in [20]. Similarly, a DF EH network consisting of one or multiple sources, multiple relays, and one destination was studied in [21], and a more general DF system consisting of a multiple source-destination pairs and multiple EH relays in the presence of random interferences was studied in [22], where the small-scale fading and the large-scale path-loss were taken into account in the outage probability analysis.

\section{B. Motivation}

Due to the far-field isotropic RF radiation, the energy transfer efficiency of WPT (e.g., microwave power transfer) via RF signals is very low. Beamforming techniques (based on large 
antenna arrays) can substantially boost the energy efficiency by forming focused energy beams using the instantaneous CSI. The existing works [2], [5]-[22] on SWIPT have either explicitly or implicitly assumed coherent WIT which requires the instantaneous CSI for information decoding. For EH relay systems featuring coherent WIT, the source needs to periodically send training symbols for channel estimation, which results in an increased network signaling overhead. Moreover, sophisticated channel estimation algorithm needs to be implemented in a distributed manner among spatially separated wireless terminals, which inevitably increases the hardware complexity and results in extra energy consumption. The consumed energy due to channel estimation and training becomes a serious issue especially for EH relay systems, as the consumed power reduces the net harvested energy for future data relaying. Therefore, coherent SWIPT requiring channel estimation might be a costly approach for energy-constrained applications. As a remedy, the noncoherent SWIPT which eliminates the need for training signalings and the channel estimation, and consequently, consumes much less energy, might be an energy-efficient and cost-effective solution for EH relay systems. To the best of our knowledge, however, the noncoherent SWIPT has not been studied in the literature, which motivated this work.

The existing works on SWIPT have been devoted to the information-theoretic performance analysis in terms of the rate-energy tradeoff, ergodic capacity, outage probability, etc. However, insights into the SWIPT regarding the bit or symbol error rate performance associated with an actual detection scheme from the communication-theoretic perspective are not available. To the best of our knowledge, the maximum-likelihood detector (MLD) achieving noncoherent SWIPT has not been studied yet. In this paper, we will tackle the noncoherent MLDs which characterize the optimum performance benchmark for ID in EH relay systems.

\section{Contribution}

The main objective of this paper is to develop the noncoherent SWIPT framework and practical MLDs for EH relay networks. We consider an EH relay system consisting of a source, a destination, and multiple EH relays, which can harvest energy directly from the RF signals radiated by the source and utilize the harvested energy to decode-and-forward the source information to the destination. The main contributions of the paper are summarized as follows:

- A unified noncoherent SWIPT framework embracing both power-splitting noncoherent DF (PS-NcDF) and time-switching noncoherent DF (TS-NcDF) protocols is developed, which 
supports any $M$-DPSK or $M$-FSK signalings enabling the noncoherent SWIPT.

- Following the unified noncoherent SWIPT framework, we derive the exact MLDs for PS$\mathrm{NcDF}$ and TS-NcDF in a unified form, for both $M$-FSK and $M$-DPSK signalings. The exact MLDs involve numerical integral evaluations; yet are useful for characterizing the optimum performance benchmark for ID in noncoherent SWIPT.

- To reduce the computational cost of the exact MLDs, we develop closed-form approximate MLDs with substantially lower complexity. Numerical results demonstrate that the proposed approximate MLDs achieve almost identical performance to the exact MLDs, thus constituting a practical solution for noncoherent SWIPT.

- Useful insights into the noncoherent SWIPT are obtained from the communication-theoretic perspective in terms of the symbol-error rate (SER). Specifically, we demonstrate a performance tradeoff between the first and second hops through the adjustment of the time switching coefficient or power splitting factor. Moreover, the optimal values of these system parameters corresponding to the minimum symbol-error rate (SER) are strictly between 0 and 1. Finally, we demonstrate that $M$-FSK is an energy-efficient solution for noncoherent SWIPT, especially when $M \geq 8$.

- As a byproduct of this work, we obtain generic analytical results dealing with the distributions of circularly symmetric complex Gaussian (CSCG) random variables and their transformations. These generic results may be of general usefulness for other different applications. Moreover, an exact expression of the transition (error) probability is developed for $M$-DPSK. In addition, a tight closed-form approximation of the transition probability is developed. To the best of our knowledge, these analytical results have not been reported in the literature.

\section{Organization and Notation}

Section III introduces the system model of EH relay networks. Section III develops the noncoherent SWIPT framework for EH relay networks. Section IV presents the mathematical fundamentals developed in this paper. Section $\mathrm{V}$ proposes the closed-form approximate as well as the exact MLDs. Section VI evaluates the performance of the proposed detectors and Section VII concludes the paper.

Notation: We use $A \triangleq B$ to denote that $A$ is defined by $B$. Also, $(\cdot)^{*},(\cdot)^{T},(\cdot)^{H},\|\cdot\|, \mathbb{E}(\cdot)$, 
and $\ln (\cdot)$ denote the conjugate, transpose, conjugate transpose, 2-norm, expectation, and natural logarithm, respectively. $\boldsymbol{I}_{n}$ denotes an $n \times n$ identity matrix, $\mathbf{0}$ denotes an all-zero column vector, $\mathbf{i}_{n}$ denotes a column vector with 1 at its $n$-th entry and 0 elsewhere, and $\measuredangle z$ denotes the phase of $z$ over a $2 \pi$ interval of interest. Finally, $\boldsymbol{x} \sim \mathcal{C N}(\boldsymbol{\mu}, \boldsymbol{\Sigma})$ indicates that $\boldsymbol{x}$ is a CSCG random vector with mean $\boldsymbol{\mu}$ and covariance $\boldsymbol{\Sigma}$.

\section{System MODEL}

Consider an EH relay system comprising a source terminal $\mathrm{T}_{0}$, a destination terminal $\mathrm{T}_{d}$, and $K$ relays $\mathrm{T}_{r}, r=1,2, \cdots, K$. The source and destination are powered by dedicated energy sources such as battery or power grid, while the relays have no power supply and instead can harvest energy from the RF signals radiated by the source. Using the harvested energy, the relays assist the source-destination communication through the noncoherent DF relaying, enabling SWIPT in EH relay systems where the instantaneous CSI is not needed.

We consider a composite fading model where the wireless channels are subject to both smallscale fading and large-scale path-loss. Note that the path-loss can lead to a serious deterioration in the power transfer efficiency and thus cannot be neglected in $\mathrm{EH}$ systems [22]. Let $\mathcal{L}_{i j}$ denote the path-loss associated with the channel from the transmit terminal $i$ to the receive terminal $j$, and $h_{i j} \sim \mathcal{C N}(0,1)$ denotes the corresponding small-scale Rayleigh fading coefficient, for $i j \in\{0 d, 0 r, r d\}_{r=1}^{K}$. Note that this channel modeling implies that the actual variances of the fading coefficients $h_{i j}$ are absorbed into the corresponding path-loss components $\mathcal{L}_{i j}$, and thus, a general asymmetric fading model is essentially considered by choosing distinct path-loss components $\mathcal{L}_{i j}$ for different wireless links. We consider the noncoherent communication where the instantaneous CSIs, $h_{i j}, i j \in\{0 d, 0 r, r d\}_{r=1}^{K}$, are unknown to all terminals in the network. The SWIPT is accomplished through the noncoherent signalings such as $M$-FSK and $M$-DPSK. Specifically, the source broadcasts RF signals according to the noncoherent $M$-FSK or $M$-DPSK modulation. The relays utilize the harvested energy to noncoherently decode and forward the information to the destination, where the ultimate noncoherent detection is performed. Thus, the end-to-end information transfer is accomplished through pure noncoherent signalings.

Due to the absence of the CSI, sophisticated protocols such as distributed space-time coding, beamforming, and relay selection which require the CSI cannot be applied. Instead, we adopt the time-division based protocol which coordinates multiple terminals' transmissions in different 
time intervals. Specifically, while the source transmits its signal, all relays stay silent and harvest energy from the source RF signals. After the source transmission, the relays decode the source information and forward it to the destination in different time intervals, allowing the destination to combine multiple received signals and thus enabling the diversity gain. Note that the timedivision relaying protocol allows the developed detectors to be readily integrated into existing time-division multiple access (TDMA) systems, and it has been considered in numerous works including EH relay systems [15], [16], [18]-[22] and self-powered relay networks [23]-[25].

For ease of the exposition, we focus on the single-antenna transmission over frequency-flat fading channels in this paper. However, the extension to the multi-antenna and/or frequencyselective fading is possible. Specifically, by applying the multi-carrier transmission such as orthogonal frequency division multiplexing (OFDM), the frequency-selective fading is automatically turned into flat fading, thus enabling the application of our scheme. Moreover, the extension to the multiple-input multiple-output (MIMO) configuration over each hop is also possible. The remaining issue is to design the noncoherent/differential space-time codes specifically suitable for EH relay systems, which may be considered as a future work.

\section{A UNIFIED NONCOHERENT SWIPT FRAMEWORK}

For EH systems, the main motivation to use noncoherent modulation schemes such as noncoherent FSK and DPSK 1 coupled with appropriate noncoherent detection schemes is that the periodic training symbols and sophisticated channel estimation algorithms are completely eliminated, which results in substantial reduction of the signaling overhead and processing burden, and consequently reduces the energy consumption in the network. In this section, by modifying the power splitting and time switching receiver structures for coherent SWIPT [2] where the instantaneous CSI is required, we develop a unified noncoherent SWIPT framework embracing both PS-NcDF and TS-NcDF requiring no instantaneous CSI.

\section{A. PS-NcDF Relaying}

In the PS-NcDF protocol, the WPT and WIT circuits at each relay terminal function simultaneously while the power fed to each circuit is a portion split from the front-end RF received

\footnotetext{
${ }^{1}$ We do not consider $M$-QAM modulation in this paper because it is typically used for coherent communication where the instantaneous CSI is available.
} 


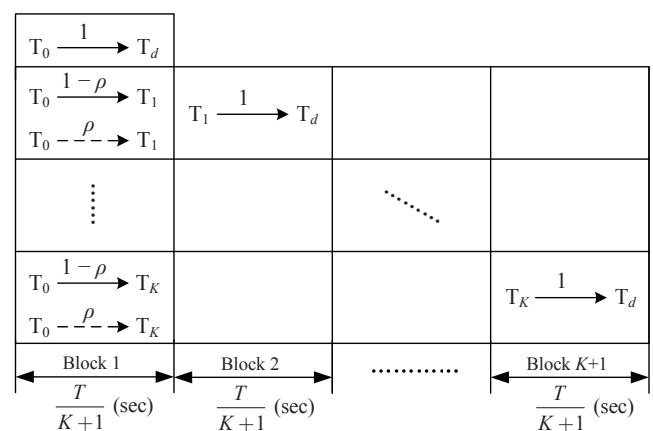

(a) PS-NcDF

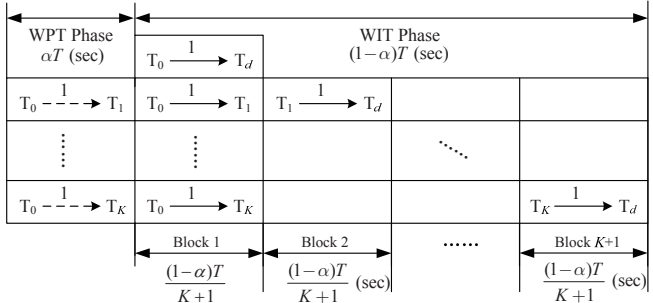

(b) $\mathrm{TS}-\mathrm{NcDF}$

Fig. 1. PS-NcDF and TS-NcDF protocols, where the solid and dashed arrow lines represent the information flow and energy flow, respectively. The numbers above the arrow lines indicate the portions of the power utilized for corresponding purposes.

signal. Let $T$ ( $\mathrm{sec}$ ) denote the total transmission time divided into $K+1$ blocks, each of which of duration $\frac{T}{K+1}(\mathrm{sec})$ is allocated to one of the transmitter including the source and $K$ relays, as illustrated in Fig. 1(a), Each block consists of $N_{s}$ symbols of duration $T_{s}(\mathrm{sec})$ each. In the first block, the source broadcasts its signal with power $P_{0}$ (watts). Each relay splits its received RF signal power into two portions: the $\rho$ portion is utilized for WPT and the harvested energy at relay $\mathrm{T}_{r}$ is $E_{r}=\eta \rho P_{0} \mathcal{L}_{0 r}\left|h_{0 r}\right|^{2} \frac{T}{K+1}, r=1, \cdots, K$, where $0<\eta<1$ is the EH efficiency [2].2 The remaining $1-\rho$ portion is for WIT. Thus, the harvested power $P_{r}$ (watts) available for data relaying at $\mathrm{T}_{r}$ is given by

$$
P_{r}=\frac{E_{r}}{\frac{T}{K+1}}=\eta \rho P_{0} \mathcal{L}_{0 r}\left|h_{0 r}\right|^{2} .
$$

In the remaining $K$ blocks, the relays take turns to decode and forward the source information to the destination using full portion of the harvested power $P_{r}$ in (1). An extreme case of $\rho=0$ represents a pure WIT system with no EH, while $\rho=1$ corresponds to a pure WPT system with no ID. In this paper, we consider a general SWIPT system featuring both WIT and WPT; thus, we have $0<\rho<1$.

1) $P S$-NcDF for $M$-DPSK: For the $M$-DPSK signaling, the source message $m \in \mathcal{M} \triangleq$ $\{0,1, \cdots, M-1\}$ in the $l$-th symbol time, $l=0,1, \cdots$, is conveyed via two consecutive information-bearing symbols $s(l)$ and $s(l-1)$ according to the differential encoding $s(l)=$ $s(l-1) \mathrm{e}^{j 2 \pi m / M}$, where $s(0)=1$ is the initial reference signal. According to the power splitting,

\footnotetext{
${ }^{2}$ Note that the inter-relay channel (IRC) is not exploited for EH, because the energy that could be harvested at a relay from any other EH relays via the IRC is negligible as compared to the energy harvested directly from the source.
} 
the received RF signal power at each relay is split in the proportion of $\rho$ and $1-\rho$, where the fractions $\rho$ and $1-\rho$ of the power are utilized for EH and ID, respectively. Thus, the received signal at $\mathrm{T}_{r}, r=1, \cdots, K$, intended for ID is

$$
y_{0 r}(l)=\sqrt{(1-\rho) P_{0} T_{s} \mathcal{L}_{0 r}} h_{0 r} s(l)+\sqrt{1-\rho} u_{0 r}(l)+v_{0 r}(l),
$$

where $T_{s}(\mathrm{sec})$ is the symbol time, $u_{0 r}(l) \sim \mathcal{C N}\left(0, \sigma_{0 r, 1}^{2}\right)$ is the AWGN introduced at the receive antenna at $\mathrm{T}_{r}$, and $v_{0 r}(l) \sim \mathcal{C N}\left(0, \sigma_{0 r, 2}^{2}\right)$ is the AWGN due to the ID circuit at $\mathrm{T}_{r}$ (which includes the RF-to-baseband conversion noise, the analog-to-digital conversion noise, circuit loss, etc) [2], [11]. The destination does not have a power splitter and the received signal is solely used for ID. Thus, the received sinal at the destination from the direct source-destination link is given by

$$
y_{0 d}(l)=\sqrt{P_{0} T_{s} \mathcal{L}_{0 d}} h_{0 d} s(l)+u_{0 d}(l)+v_{0 d}(l)
$$

where $u_{0 d}(l) \sim \mathcal{C N}\left(0, \sigma_{0 d, 1}^{2}\right)$ and $v_{0 d}(l) \sim \mathcal{C N}\left(0, \sigma_{0 d, 2}^{2}\right)$ are the AWGNs due to the receive antenna and ID circuit at $\mathrm{T}_{d}$, respectively. Each relay node assists the communication from the source to the destination via the DF protocol, where $\mathrm{T}_{r}$ detects the source message by ML detection and forwards the detected message $m_{r} \in \mathcal{M}$ with full harvested power $P_{r}$ in (1) to the destination in a time-division manner via the $M$-DPSK transmission. Specifically, the transmitted symbols $s_{r}(l), l=0,1, \cdots$, at $\mathrm{T}_{r}$ are successively related according to the differential encoding $s_{r}(l)=s_{r}(l-1) \mathrm{e}^{j 2 \pi m_{r} / M}$, where $s_{r}(0)=1$ is the initial reference signal at $\mathrm{T}_{r}, r=1, \cdots, K$. The signal received at the destination from $\mathrm{T}_{r}$ is given by

$$
y_{r d}(l)=\sqrt{P_{r} T_{s} \mathcal{L}_{r d}} h_{r d} s_{r}(l)+u_{r d}(l)+v_{r d}(l),
$$

where $u_{r d}(l) \sim \mathcal{C N}\left(0, \sigma_{r d, 1}^{2}\right)$ and $v_{r d}(l) \sim \mathcal{C N}\left(0, \sigma_{r d, 2}^{2}\right)$ are AWGNs due to the receive antenna and ID circuit, respectively. It is assumed that all AWGNs are mutually independent.

2) $P S-N c D F$ for $M$-FSK: For noncoherent $M$-FSK, the source message $m \in \mathcal{M}$ is transmitted over one of the $M$ orthogonal carriers. The received RF signal at each receive terminal is fed to $M$ orthogonal subband correlators, yielding an $M \times 1$ baseband equivalent signal vector $\mathbf{y}_{i j} \triangleq\left[\mathrm{y}_{i j}(1), \cdots, \mathrm{y}_{i j}(M)\right]^{T}, i j \in\{0 d, 0 r, r d\}_{r=1}^{N}$. The baseband equivalent signal received at the destination from the direct link is

$$
\mathbf{y}_{0 d}=\sqrt{P_{0} T_{s} \mathcal{L}_{0 d}} h_{0 d} \mathbf{i}_{m+1}+\mathbf{u}_{0 d}+\mathbf{v}_{0 d},
$$


where $\mathbf{u}_{0 d} \sim \mathcal{C N}\left(\mathbf{0}, \sigma_{0 d, 1}^{2} \boldsymbol{I}_{M}\right)$ and $\mathbf{v}_{0 d} \sim \mathcal{C N}\left(\mathbf{0}, \sigma_{0 d, 2}^{2} \boldsymbol{I}_{M}\right)$ are the AWGNs due to the receive antenna and the ID circuit at $\mathrm{T}_{d}$, respectively. Due to power splitting at each relay, the received signal intended for ID at $\mathrm{T}_{r}$ is scaled by the factor $\sqrt{1-\rho}$, and the baseband equivalent received signal at $\mathrm{T}_{r}, r=1, \cdots, K$, is given by

$$
\mathbf{y}_{0 r}=\sqrt{(1-\rho) P_{0} T_{s} \mathcal{L}_{0 r}} h_{0 r} \mathbf{i}_{m+1}+\sqrt{1-\rho} \mathbf{u}_{0 r}+\mathbf{v}_{0 r},
$$

where $\mathbf{u}_{0 r} \sim \mathcal{C N}\left(\mathbf{0}, \sigma_{0 r, 1}^{2} \boldsymbol{I}_{M}\right)$ and $\mathbf{v}_{0 r} \sim \mathcal{C N}\left(\mathbf{0}, \sigma_{0 r, 2}^{2} \boldsymbol{I}_{M}\right)$ are AWGNs due to the receive antenna and the ID circuit at $\mathrm{T}_{r}$, respectively. Each relay decodes the source message and forwards the decoded message $m_{r} \in \mathcal{M}$ with full power $P_{r}$ in (1) to the destination in a time-division fashion. The baseband equivalent signal received at the destination from $\mathrm{T}_{r}$ is given by

$$
\mathbf{y}_{r d}=\sqrt{P_{r} T_{s} \mathcal{L}_{r d}} h_{r d} \mathbf{i}_{m_{r}+1}+\mathbf{u}_{r d}+\mathbf{v}_{r d}
$$

where $\mathbf{u}_{r d} \sim \mathcal{C N}\left(\mathbf{0}, \sigma_{r d, 1}^{2} \boldsymbol{I}_{M}\right)$ and $\mathbf{v}_{r d} \sim \mathcal{C N}\left(\mathbf{0}, \sigma_{r d, 2}^{2} \boldsymbol{I}_{M}\right)$ are AWGNs due to receive antenna and ID circuit at $\mathrm{T}_{d}$, respectively. The AWGNs are assumed mutually independent.

\section{B. TS-NcDF Relaying}

In the TS-NcDF protocol, the WPT and WIT functionalities take place in a time-division fashion. Specifically, the total transmission time $T$ (sec) is divided into two phases: the WPT phase of duration $\alpha T$ and the WIT phase of duration $(1-\alpha) T$, as illustrated in Fig. 1(b). In the WPT phase, the source radiates RF energy flow with power $P_{0}$ to the relays, where only the EH circuits are activated. Thus, the harvested energy at $\mathrm{T}_{r}$ is $E_{r}=\eta P_{0} \mathcal{L}_{0 r}\left|h_{0 r}\right|^{2} \alpha T$, $r=1, \cdots, K$, where $0<\eta<1$ is the energy conversion efficiency [2]. In the WIT phase, only the ID circuit is activated at each relay. To coordinate multiple terminals' transmissions, the WIT phase is further divided into $K+1$ blocks of duration $\frac{(1-\alpha) T}{K+1}$ each. In the first block, the source broadcasts information with power $P_{0}$ to all relays. The relay $\mathrm{T}_{r}$ utilizes its harvested energy $E_{r}$ to decode and forward the source information to the destination in the $(r+1)$-th block, $r=1, \cdots, K$. Thus, the harvested power $P_{r}$ available for data relaying at $\mathrm{T}_{r}$ is given by

$$
P_{r}=\frac{E_{r}}{\frac{(1-\alpha) T}{K+1}}=\frac{(K+1) \eta P_{0} \mathcal{L}_{0 r}\left|h_{0 r}\right|^{2} \alpha}{1-\alpha} .
$$

An extreme case of $\alpha=0$ represents a pure WIT system with no $\mathrm{EH}$, and $\alpha=1$ corresponds to a pure WPT system with no ID. In this paper, we consider $0<\alpha<1$, which corresponds to the general SWIPT relay system featuring both WIT and WPT. 
1) TS-NcDF for M-DPSK: In the TS-NcDF protocol, the signal received at each terminal during the WIT phase is solely utilized for ID, i.e., no power splitting is involved. Consequently, the signal model for TS-NcDF employing the $M$-DPSK is depicted as follows:

$$
\begin{aligned}
& y_{0 d}(l)=\sqrt{P_{0} T_{s} \mathcal{L}_{0 d}} h_{0 d} s(l)+u_{0 d}(l)+v_{0 d}(l), \\
& y_{0 r}(l)=\sqrt{P_{0} T_{s} \mathcal{L}_{0 r}} h_{0 r} s(l)+u_{0 r}(l)+v_{0 r}(l), \\
& y_{r d}(l)=\sqrt{P_{r} T_{s} \mathcal{L}_{r d}} h_{r d} s_{r}(l)+u_{r d}(l)+v_{r d}(l),
\end{aligned}
$$

$r=1, \cdots, K$, where $u_{i j}(l) \sim \mathcal{C N}\left(0, \sigma_{i j, 1}^{2}\right)$ and $v_{i j}(l) \sim \mathcal{C N}\left(0, \sigma_{i j, 2}^{2}\right)$ are AWGNs due to the receive antenna and ID circuit, respectively, ij $\in\{0 d, 0 r, r d\}_{r=1}^{K}$. The source symbol $s(l)$ is related to the message $m$ through $s(l)=s(l-1) \mathrm{e}^{j 2 \pi m / M}$. Similarly, $s_{r}(l)=s_{r}(l-1) \mathrm{e}^{j 2 \pi m_{r} / M}$.

2) TS-NcDF for M-FSK: The signal model for TS-NcDF employing noncoherent $M$-FSK is

$$
\begin{aligned}
& \mathbf{y}_{0 d}=\sqrt{P_{0} T_{s} \mathcal{L}_{0 d}} h_{0 d} \mathbf{i}_{m+1}+\mathbf{u}_{0 d}+\mathbf{v}_{0 d}, \\
& \mathbf{y}_{0 r}=\sqrt{P_{0} T_{s} \mathcal{L}_{0 r}} h_{0 r} \mathbf{i}_{m+1}+\mathbf{u}_{0 r}+\mathbf{v}_{0 r}, \\
& \mathbf{y}_{r d}=\sqrt{P_{r} T_{s} \mathcal{L}_{r d}} h_{r d} \mathbf{i}_{m_{r}+1}+\mathbf{u}_{r d}+\mathbf{v}_{r d},
\end{aligned}
$$

for $r=1, \cdots, K$, where $\mathbf{u}_{i j} \sim \mathcal{C N}\left(\mathbf{0}, \sigma_{i j, 1}^{2} \boldsymbol{I}_{M}\right)$ and $\mathbf{v}_{i j} \sim \mathcal{C N}\left(\mathbf{0}, \sigma_{i j, 2}^{2} \boldsymbol{I}_{M}\right), i j \in\{0 d, 0 r, r d\}_{r=1}^{K}$.

\section{A Unified Noncoherent SWIPT Framework}

Since we consider two protocols, namely PS-NcDF and TS-NcDF, employing two different noncoherent modulations based on $M$-DPSK or $M$-FSK, there are in total four possible schemes considering different combinations, which make the derivations of the detectors rather tedious. For ease of development, we now unify the system models of PS-NcDF and TS-NcDF such that the detectors for PS-NcDF and TS-NcDF are derived in a general unified form. The key to the unifying process is a set of reparameterizations of the noise variances and the signal-to-noise ratios (SNRs) as follows:

$$
\begin{gathered}
\gamma_{0 d} \triangleq \frac{P_{0} T_{s} \mathcal{L}_{0 d}}{\sigma_{0 d, 1}^{2}+\sigma_{0 d, 2}^{2}}, \quad \sigma_{k d}^{2} \triangleq \sigma_{k d, 1}^{2}+\sigma_{k d, 2}^{2}, \quad \sigma_{0 r}^{2} \triangleq \begin{cases}(1-\rho) \sigma_{0 r, 1}^{2}+\sigma_{0 r, 2}^{2}, & \text { PS-NcDF, } \\
\sigma_{0 r, 1}^{2}+\sigma_{0 r, 2}^{2}, & \text { TS-NcDF },\end{cases} \\
\gamma_{0 r} \triangleq\left\{\begin{array} { l l } 
{ \frac { ( 1 - \rho ) P _ { 0 } T _ { s } \mathcal { L } _ { 0 r } } { ( 1 - \rho ) \sigma _ { r r , 1 } ^ { 2 } + \sigma _ { 0 r , 2 } ^ { 2 } } , } & { \text { PS-NcDF, } } \\
{ \frac { P _ { 0 } T _ { 0 } \mathcal { L } _ { 0 r } } { \sigma _ { 0 r , 1 } ^ { 2 } + \sigma _ { 0 r , 2 } ^ { 2 } } , } & { \text { TS-NcDF, } }
\end{array} \gamma _ { r d } \triangleq \left\{\begin{array}{ll}
\frac{\eta \rho P_{0} T_{s} \mathcal{L}_{0 r} \mathcal{L}_{r d}}{\sigma_{r d, 1}^{2}+\sigma_{r d, 2}^{2},} & \text { PS-NcDF, } \\
\frac{\alpha(K+1) \eta P_{0} T_{s} \mathcal{L}_{0 r} \mathcal{L}_{r d},}{(1-\alpha)\left(\sigma_{r d, 1}^{2}+\sigma_{r d, 2}^{2}\right)}, & \text { TS-NcDF, }
\end{array}\right.\right.
\end{gathered}
$$

where $r=1, \cdots, K$ and $k=0, \cdots, K$. 
1) Unified PS/TS-NcDF Framework for M-DPSK: In the $M$-DPSK transmission, each message is conveyed via two consecutive symbols. Thus, it is convenient to represent two consecutive received symbols as a vector. To that end, we introduce the following vectors:

$$
\boldsymbol{y}_{i j} \triangleq\left[\begin{array}{c}
y_{i j}(l-1) \\
y_{i j}(l)
\end{array}\right], \quad \boldsymbol{s} \triangleq\left[\begin{array}{c}
s(l-1) \\
s(l)
\end{array}\right], \quad \boldsymbol{s}_{r} \triangleq\left[\begin{array}{c}
s_{r}(l-1) \\
s_{r}(l)
\end{array}\right] \text {. }
$$

Using definitions in (11), we can unify PS-NcDF and TS-NcDF for $M$-DPSK as follows:

$$
\begin{aligned}
& \boldsymbol{y}_{0 r}=\sigma_{0 r}\left(\sqrt{\gamma_{0 r}} h_{0 r} \boldsymbol{s}+\boldsymbol{n}_{0 r}\right), \\
& \boldsymbol{y}_{0 d}=\sigma_{0 d}\left(\sqrt{\gamma_{0 d}} h_{0 d} \boldsymbol{s}+\boldsymbol{n}_{0 d}\right), \\
& \boldsymbol{y}_{r d}=\sigma_{r d}\left(\sqrt{\gamma_{r d}} h_{0 r} \mid h_{r d} \boldsymbol{s}_{r}+\boldsymbol{n}_{r d}\right),
\end{aligned}
$$

where $\boldsymbol{n}_{i j} \sim \mathcal{C N}\left(\mathbf{0}, \boldsymbol{I}_{2}\right)$ for $i j \in\{0 d, 0 r, r d\}_{r=1}^{K}$. By appropriately choosing the parameters according to (11), the unified model (13) can represent either PS-NcDF or TS-NcDF.

2) Unified PS/TS-NcDF Framework for Noncoherent M-FSK: Based on the parameters in (11), the system models of PS-NcDF and TS-NcDF associated with noncoherent $M$-FSK can be expressed in a unified form as follows:

$$
\begin{aligned}
& \mathbf{y}_{0 r}=\sigma_{0 r}\left(\sqrt{\gamma_{0 r}} h_{0 r} \mathbf{i}_{m+1}+\mathbf{n}_{0 r}\right), \\
& \mathbf{y}_{0 d}=\sigma_{0 d}\left(\sqrt{\gamma_{0 d}} h_{0 d} \mathbf{i}_{m+1}+\mathbf{n}_{0 d}\right), \\
& \mathbf{y}_{r d}=\sigma_{r d}\left(\sqrt{\gamma_{r d}}\left|h_{0 r}\right| h_{r d} \mathbf{i}_{m_{r}+1}+\mathbf{n}_{r d}\right),
\end{aligned}
$$

where $\mathbf{n}_{i j} \sim \mathcal{C N}\left(\mathbf{0}, \boldsymbol{I}_{M}\right)$ for $i j \in\{0 d, 0 r, r d\}$ and $r=1, \cdots, K$. For ease of readability, a list of symbols are summarized in Table I,

Under the noncoherent SWIPT framework, the instantaneous CSIs $h_{i j}, i j \in\{0 d, 0 r, r d\}_{r=0}^{K}$, are not available to the whole network, which makes the noncoherent detection necessary. To ensure the end-to-end optimality of the noncoherent communication, it is required that the destination as well as the relays perform the ML detection. Specifically, each relay performs its own (noncoherent) ML detection individually and forwards its estimated message to the destination. Due to potential decoding errors at the relays, the ultimate (noncoherent) ML detection at the destination must take into account the transition decoding error probabilities in all the relays, which will be treated in more detail in what follows. 
TABLE I

LIST OF SYMBOLS.

\begin{tabular}{|c|c||c|c|}
\hline$M$ & Modulation alphabet size & $P_{r}, E_{r}$ & Harvested power and energy at the $r$-th relay \\
\hline$K$ & Number of relays & $T_{s}, T$ & Symbol time and block time \\
\hline$\eta$ & Energy conversion efficiency & $\mathrm{T}_{i}$ & The $i$-th terminal \\
\hline$\alpha$ & Time switching coefficient & $h_{i j}, \mathcal{L}_{i j}$ & Channel coefficient, path loss for the $\mathrm{T}_{i}$ - $\mathrm{T}_{j}$ link \\
\hline$\rho$ & Power splitting factor & $\sigma_{i j, 1}^{2}, \sigma_{i j, 2}^{2}$ & Variances of AWGNs due to receive antenna and ID circuit \\
\hline$P_{0}$ & Source transmission power & $\sigma_{i j}^{2}, \gamma_{i j}$ & Noise variance and SNR of $\mathrm{T}_{i}-\mathrm{T}_{j}$ link \\
\hline
\end{tabular}

\section{Mathematical Fundamentals}

To facilitate the development of the noncoherent detection schemes, it is necessary to study the distributions involving combinations of CSCG and Rayleigh random variables/vectors, as indicated by the unified SWIPT frameworks in (13) and (14).

\section{A. Generic Probability Analysis}

We carry out a generic probability analysis involving independent random varaibles/vectors as follows:

$$
\begin{aligned}
\boldsymbol{x}_{0} & \sim \mathcal{C N}\left(\mathbf{0}, \Omega_{0} \boldsymbol{I}_{2}\right), \quad \boldsymbol{y}_{0} \sim \mathcal{C N}\left(\mathbf{0}, \Omega_{0} \boldsymbol{I}_{M}\right), \\
X_{i} & \sim \mathcal{C N}\left(0, \Omega_{i}\right), i=1,2 .
\end{aligned}
$$

We are interested in the following random vectors:

$$
\begin{gathered}
\boldsymbol{X}_{0} \triangleq X_{1}\left|X_{2}\right| \boldsymbol{c}+\boldsymbol{x}_{0}, \\
\boldsymbol{Y}_{0} \triangleq X_{1}\left|X_{2}\right| \mathbf{i}_{p}+\boldsymbol{y}_{0},
\end{gathered}
$$

where $c \triangleq[1, c]^{T}, c$ is any complex constant, and $1 \leq p \leq M$ is any integer number. Note that (16) and (17) are generic forms of $\boldsymbol{y}_{r d}$ in (13c) for $M$-DPSK and $\mathbf{y}_{r d}$ in (14c) for $M$-FSK, respectively.

Lemma $1(C S C G \times$ Rayleigh $+C S C G)$ : Given the random variables/vectors in (15), the PDFs of $\boldsymbol{X}_{0}$ in (16) and $\boldsymbol{Y}_{0}$ in (17) are, respectively, given by

$$
\begin{aligned}
f_{\boldsymbol{X}_{0}}(\boldsymbol{x}) & =\frac{\exp \left(-\frac{\left|x_{2}-c x_{1}\right|^{2}}{\Omega_{0}\left(1+|c|^{2}\right)}\right)}{\pi^{2} \Omega_{0}^{2}} I\left(\frac{\Omega_{1} \Omega_{2}}{\Omega_{0}}\left(1+|c|^{2}\right), \frac{\left|x_{1}+c^{*} x_{2}\right|^{2}}{\Omega_{0}\left(1+|c|^{2}\right)}\right), \\
f_{\boldsymbol{Y}_{0}}(\boldsymbol{y}) & =\frac{1}{\left(\pi \Omega_{0}\right)^{M}} \mathrm{e}^{-\frac{\|\boldsymbol{y}\|^{2}-\left|y_{p}\right|^{2}}{\Omega_{0}}} I\left(\frac{\Omega_{1} \Omega_{2}}{\Omega_{0}}, \frac{\left|y_{p}\right|^{2}}{\Omega_{0}}\right),
\end{aligned}
$$


where $\boldsymbol{x} \triangleq\left[x_{1}, x_{2}\right]^{T}, \boldsymbol{y} \triangleq\left[y_{1}, \cdots, y_{M}\right]^{T}$, and $I(\cdot, \cdot)$ is defined as

$$
I(\epsilon, \beta) \triangleq \frac{\mathrm{e}^{\frac{1}{\epsilon}}}{\epsilon} \int_{0}^{\beta} \frac{1}{x} \mathrm{e}^{-\left(x+\frac{\beta}{\epsilon x}\right)} \mathrm{d} x
$$

Proof: See Appendix A.

Corollary $1(C S C G \times C S C G+C S C G)$ : Let $\boldsymbol{X}_{1} \triangleq X_{1} X_{2} \boldsymbol{c}+\boldsymbol{x}_{0}$ and $\boldsymbol{Y}_{1} \triangleq X_{1} X_{2} \mathbf{i}_{p}+\boldsymbol{y}_{0}$. Then the PDF of $\boldsymbol{X}_{1}, f_{\boldsymbol{X}_{1}}(\boldsymbol{x})$, and the PDF of $\boldsymbol{Y}_{1}, f_{\boldsymbol{Y}_{1}}(\boldsymbol{y})$, are given by $f_{\boldsymbol{X}_{1}}(\boldsymbol{x})=f_{\boldsymbol{X}_{0}}(\boldsymbol{x})$ and $f_{\boldsymbol{Y}_{1}}(\boldsymbol{y})=f_{\boldsymbol{Y}_{0}}(\boldsymbol{y})$.

Proof: Due to rotational invariance of the CSCG distribution [26], it can be shown that $X_{1} X_{2}$ follows the same distribution as $X_{1}\left|X_{2}\right|$, which yields the above result.

A comprehensive study of the distribution of the product of two independent CSCG random variables, namely, "CSCG $\times$ CSCG", was also presented in [27]. However, the result in [27] was given in terms of modified bessel function. If one utilizes the result of [27], then the PDF of "CSCG $\times$ CSCG + CSCG" may be derived as an infinite convolution integral of the modified bessel function with the Gaussian PDF, which is prohibitively more complex than the result obtained here. Note that the finite integral of (20) has a well-behaved integrand involving only elementary functions, and it has a fast convergence speed which allows for fast evaluations at very high accuracy, as will be demonstrated later.

In order to develop the MLD at the destination, the transition (error) probability of the DPSK detector at the relay needs to be determined. To that end, it is useful to study the distribution of the phase difference of two consecutively received DPSK signals.

Lemma 2: Given the random variables/vectors in (15), we consider the $2 \times 1$ random vector $\boldsymbol{Y}_{2} \triangleq\left[Y_{1}, Y_{2}\right]^{T}=X_{1} \boldsymbol{c}+\boldsymbol{x}_{0}$, where $\boldsymbol{c}=[1, c]^{T}$. Then the PDF of $\Theta \triangleq \measuredangle Y_{1}^{*} Y_{2}-\measuredangle c$ is

$$
f_{\Theta}(\theta)=\frac{1}{2 \pi} \frac{1}{1+\frac{\gamma^{2}|c|^{2} \sin ^{2} \theta}{1+\gamma\left(1+|c|^{2}\right)}}\left[1+\frac{\gamma|c| \cos \theta \arccos \left(-\frac{\gamma|c| \cos \theta}{\sqrt{\gamma^{2}|c|^{2}+\gamma\left(1+|c|^{2}\right)+1}}\right)}{\sqrt{\gamma^{2}|c|^{2} \sin ^{2} \theta+\gamma\left(1+|c|^{2}\right)+1}}\right],
$$

where $\gamma \triangleq \Omega_{1} / \Omega_{0}$ and $\theta$ is defined in any $2 \pi$ interval of interest 3

Proof: See Appendix B.

The phase difference distribution of PSK/DPSK signals was studied for a simplistic AWGN channel with no fading [28], where the PDF was given in an integral form. In contrast, in addition

\footnotetext{
${ }^{3}$ Mathematically, $f_{\Theta}(\theta)$ in 21 is a periodic function with period $2 \pi$. Thus, one may consider the domain of $\theta$ as any $2 \pi$ interval of interest such as $[0,2 \pi)$ or $[-\pi, \pi)$.
} 
to the AWGN $\boldsymbol{x}_{0} \sim \mathcal{C N}\left(\mathbf{0}, \Omega_{0} \boldsymbol{I}_{2}\right)$, we impose Rayleigh fading where the fading coefficient is modeled by $X_{1} \sim \mathcal{C N}\left(0, \Omega_{1}\right)$. For the fading scenario, the obtained PDF is given by a truly closed-form expression (21), which is more suitable for further analysis.

The results in Lemma 1 and Corollary 1 provide a useful characterization of the joint magnitude and phase distribution of sophisticated Gaussian transformations, while the result in Lemma 2 characterizes the distribution of the input-output phase difference involving Gaussian random variables/vectors. These generic analytical results which have not been reported in the literature may be useful in a different context involving different applications.

\section{B. Numerical Computation of $I(\epsilon, \beta)$ in (20)}

To facilitate the evaluation of $I(\epsilon, \beta)$ in (20), we now derive some alternative expressions of $I(\epsilon, \beta)$, which are suitable for numerical computations.

Lemma 3 (Alternative Forms): $I(\epsilon, \beta)$ in (20) can be equivalently expressed as

$$
\begin{aligned}
I(\epsilon, \beta) & =\int_{0}^{\infty} \frac{1}{1+\epsilon t} \exp \left(-\frac{\beta}{1+\epsilon t}\right) \exp (-t) \mathrm{d} t \\
& = \begin{cases}\frac{\mathrm{e}^{\frac{1}{\epsilon}}}{\epsilon}\left[K_{0}\left(2 \sqrt{\frac{\beta}{\epsilon}}\right)-\int_{\beta}^{\sqrt{\frac{\beta}{\epsilon}}} \frac{1}{x} \mathrm{e}^{-\left(x+\frac{\beta}{\epsilon x}\right)} \mathrm{d} x\right], & \beta \epsilon<1, \\
\beta \mathrm{e}^{\beta} K_{0}(2 \beta), & \beta \epsilon=1, \\
\frac{\mathrm{e}^{\frac{1}{\epsilon}}}{\epsilon}\left[K_{0}\left(2 \sqrt{\frac{\beta}{\epsilon}}\right)+\int_{\frac{1}{\epsilon}}^{\sqrt{\frac{\beta}{\epsilon}}} \frac{1}{x} \mathrm{e}^{-\left(x+\frac{\beta}{\epsilon x}\right)} \mathrm{d} x\right], & \beta \epsilon>1,\end{cases}
\end{aligned}
$$

where $K_{0}(x)$ is the zero-order modified Bessel function of the second kind [29, eq. (8.432.1)].

Proof: For $\beta \epsilon<1$, (20) can be rewritten as $I(\epsilon, \beta)=\frac{\mathrm{e}^{\frac{1}{\epsilon}}}{\epsilon}\left[\int_{0}^{\sqrt{\frac{\beta}{\epsilon}}} \frac{1}{x} \mathrm{e}^{-\left(x+\frac{\beta}{\epsilon x}\right)} \mathrm{d} x-\int_{\beta}^{\sqrt{\frac{\beta}{\epsilon}}} \frac{1}{x} \mathrm{e}^{-\left(x+\frac{\beta}{\epsilon x}\right)} \mathrm{d} x\right]$. Similarly, $I(\epsilon, \beta)=\frac{\mathrm{e}^{\frac{1}{\epsilon}}}{\epsilon}\left[\int_{0}^{\sqrt{\frac{\beta}{\epsilon}}} \frac{1}{x} \mathrm{e}^{-\left(x+\frac{\beta}{\epsilon x}\right)} \mathrm{d} x+\int_{\sqrt{\frac{\beta}{\epsilon}}}^{\beta} \frac{1}{x} \mathrm{e}^{-\left(x+\frac{\beta}{\epsilon x}\right)} \mathrm{d} x\right]$ for $\beta \epsilon>1$. For $\beta \epsilon=1$, we have $I(\epsilon, \beta)=\beta \mathrm{e}^{\beta} \int_{0}^{\beta} \frac{1}{x} \mathrm{e}^{-\left(x+\frac{\beta^{2}}{x}\right)} \mathrm{d} x$. These expressions have a common integral-form $\int_{0}^{a} \frac{1}{x} \mathrm{e}^{-\left(x+\frac{a^{2}}{x}\right)} \mathrm{d} x$ with $a>0$. By change of variable, it can be shown that $\int_{0}^{a} \frac{1}{x} \mathrm{e}^{-\left(x+\frac{a^{2}}{x}\right)} \mathrm{d} x=\int_{a}^{\infty} \frac{1}{x} \mathrm{e}^{-\left(x+\frac{\left.a^{2}\right)}{x}\right)} \mathrm{d} x$. Thus, we have $\int_{0}^{a} \frac{1}{x} \mathrm{e}^{-\left(x+\frac{a^{2}}{x}\right)} \mathrm{d} x=\frac{1}{2} \int_{0}^{\infty} \frac{1}{x} \mathrm{e}^{-\left(x+\frac{a^{2}}{x}\right)} \mathrm{d} x=K_{0}(2 a)$, followed by [29, eq. (3.471.9)]. Finally, substituting $a=\sqrt{\beta / \epsilon}$ for $\beta \epsilon>1$ or $\beta \epsilon<1$ and $a=\beta$ for $\beta \epsilon=1$ yields (23).

The alternative form in (22) indicates that the original integral of (20) can be seen as the expectation of the random transformation $\frac{1}{1+\epsilon t} \exp \left(-\frac{\beta}{1+\epsilon t}\right)$, where $t$ is exponentially distributed with unit mean. The alternative form in (23) has a closed-form solution only for $\beta \epsilon=1$. For $\beta \epsilon \neq 1$, we are able to boil down the original integral into two terms, where the first term is 
given in an exact closed-form while the second term is given by a sub-integral. The benefit of using this alternative form is that the sub-integral is taken over a smaller integral interval and thus can be more easily computed as compared to the original integral in (20). We now exploit these alternative forms to derive a very useful piecewise approximation for the original integral with very high accuracy.

Lemma 4: For $\beta \epsilon \neq 1, I(\epsilon, \beta)$ in (20) can be approximated by the $N$-th order expression, $I(\epsilon, \beta)=I_{N}(\epsilon, \beta)+R_{N}, N \geq 1$, where $R_{N}$ is the $N$-th remainder and $I_{N}(\epsilon, \beta)$ is given by

$$
I_{N}(\epsilon, \beta) \triangleq \begin{cases}\tau \mathrm{e}^{-\beta \tau}\left[1+\sum_{n=2}^{N}(-1)^{n} \epsilon^{n} \tau^{n} \mu_{n} \sum_{\substack{\sum_{i=1}^{n} i m_{i}=n \\ \forall m_{i} \in \mathbb{Z}^{+}}} \frac{(\varsigma-\beta \tau)(-\beta \tau)^{\varsigma-1}}{\prod_{j=1}^{n} m_{j} !}\right], & \beta \epsilon<1, \frac{\mathrm{e}^{\frac{1}{\epsilon}}}{\epsilon}>1, \\ \frac{\mathrm{e}^{\frac{1}{\epsilon}}}{\epsilon}\left[K_{0}\left(2 \sqrt{\frac{\beta}{\epsilon}}\right)-\Psi_{N}\left(\beta, \sqrt{\frac{\beta}{\epsilon}}\right)\right], & \beta \epsilon<1, \frac{\mathrm{e}^{\frac{1}{\epsilon}}}{\epsilon} \leq 1, \\ \frac{\mathrm{e}^{\frac{1}{\epsilon}}}{\epsilon}\left[K_{0}\left(2 \sqrt{\frac{\beta}{\epsilon}}\right)+\Psi_{N}\left(\frac{1}{\epsilon}, \sqrt{\frac{\beta}{\epsilon}}\right)\right], & \beta \epsilon>1,\end{cases}
$$

where $\tau \triangleq \frac{1}{\epsilon+1}, \varsigma \triangleq \sum_{i=1}^{n} m_{i}, \mu_{n} \triangleq \sum_{i=0}^{n} \frac{(-1)^{i}}{i !} n !$, and $\mathbb{Z}^{+} \triangleq\{0,1,2, \cdots\}$. Also, $\Psi_{N}(\cdot, \cdot)$ is given by

$$
\Psi_{N}(a, b) \triangleq \frac{b-a}{2} \sum_{i=1}^{N} w_{i} \psi\left(\frac{b-a}{2} z_{i}+\frac{a+b}{2}\right)
$$

where $w_{i}$ and $z_{i}, i=1, \cdots, N$, are, respectively, the weights and zeros of the Legendre polynomial of order $N$ [30, Table 25.4], and $\psi(x) \triangleq \frac{1}{x} \mathrm{e}^{-\left(x+\frac{\beta}{\epsilon x}\right)}$.

Proof: See Appendix C.

The first piece of (24) is a direct consequence of (22) followed by the Taylor series expansion of $\frac{1}{1+\epsilon \boldsymbol{t}} \exp \left(-\frac{\beta}{1+\epsilon \boldsymbol{t}}\right)$ around the mean $\mathbb{E}[\boldsymbol{t}]=1$. The second and third pieces of (24) are direct consequences of the Gaussian-Legendre quadrature carried out for the sub-integrals in (23). Note that the closed-form piecewise approximation of $I(\epsilon, \beta)$ by means of $I_{N}(\epsilon, \beta)$ in (24) is valid for any natural number $N \geq 1$, and an improved accuracy is expected by increasing the order $N$. However, from the computational cost point-of-view, it is desirable to choose a fairly small $\operatorname{order} N$ which attains the required accuracy. To that end, we set $N=2$ to guarantee extremely 
low computational complexity, and the expression of (24) reduces to

$$
I_{2}(\epsilon, \beta)= \begin{cases}\tau \mathrm{e}^{-\beta \tau}\left(1+\epsilon^{2} \tau^{2}-2 \epsilon^{2} \tau^{3} \beta-\frac{1}{2} \epsilon^{2} \tau^{4} \beta^{2}\right), & \beta \epsilon<1, \frac{\mathrm{e}^{\frac{1}{\epsilon}}}{\epsilon}>1, \\ \frac{\mathrm{e}^{\frac{1}{\epsilon}}}{\epsilon}\left[K_{0}\left(2 \sqrt{\frac{\beta}{\epsilon}}\right)-\Psi_{2}\left(\beta, \sqrt{\frac{\beta}{\epsilon}}\right)\right], & \beta \epsilon<1, \frac{\mathrm{e}^{\frac{1}{\epsilon}}}{\epsilon} \leq 1, \\ \frac{\mathrm{e}^{\frac{1}{\epsilon}}}{\epsilon}\left[K_{0}\left(2 \sqrt{\frac{\beta}{\epsilon}}\right)+\Psi_{2}\left(\frac{1}{\epsilon}, \sqrt{\frac{\beta}{\epsilon}}\right)\right], & \beta \epsilon>1,\end{cases}
$$

where we have $z_{1}=\frac{\sqrt{3}}{3}, z_{2}=-\frac{\sqrt{3}}{3}$, and $w_{1}=w_{2}=1$ for $N=2$. Through extensive evaluations in Section VI, we will demonstrate that the piecewise approximation $I_{2}(\epsilon, \beta)$ in (26) is indeed very accurate for a wide range of $\epsilon$ and $\beta$ values. Considering its very low computational cost, we will utilize $I_{2}(\epsilon, \beta)$ in the next section to facilitate the implementation of the approximate MLDs.

\section{MLDS FOR ENERGy HARVESTING DF NETWORKS}

Following the unified noncoherent SWIPT framework in Section III, we will develop unified noncoherent detectors in this section. Specifically, we begin by finding the exact MLDs at each relay, followed by the transition probability analysis. Finally, the exact MLDs as well as the closed-form approximate MLDs at the destination are derived for both $M$-DPSK and $M$-FSK.

\section{A. Unified MLDs at the Relays}

From the unified first-hop model in (13a) and (14a), we see that the input-output relation is equivalent to the conventional P2P communication in Rayleigh fading. Thus, the MLD at the relay can be derived similar to that for P2P communication. Specifically, the MLDs of $M$-DPSK at $\mathrm{T}_{r}, r=1, \cdots, K$, for PS-NcDF and TS-NcDF are expressed in a unified form [31, Ch. 5.2.8]

$$
m_{r}=\arg \max _{m=0, \cdots, M-1} \Re\left\{y_{0 r}^{*}(l) y_{0 r}(l-1) \mathrm{e}^{j 2 \pi m / M}\right\} .
$$

Similarly, the MLDs of $M$-FSK at $\mathrm{T}_{r}$ for PS-NcDF and TS-NcDF are given by [31, Ch. 5.4.2]

$$
m_{r}=\arg \max _{m=0, \cdots, M-1}\left|\mathrm{y}_{0 r}(m+1)\right|^{2} .
$$




\section{B. Transition Probability Analysis}

Due to potential detection errors at the relay, the detected message $m_{r}$ at $\mathrm{T}_{r}$ may be different from the actual source transmitted message $m$. In fact, a potential decision error at $\mathrm{T}_{r}$ may transform the correct message $m \in \mathcal{M}$ to any incorrect message $m_{r} \in \mathcal{M}$. Thus, the transition probability, $\operatorname{Pr}\left(m_{r} \mid m\right)$, namely the probability that the message $m_{r}$ is detected while $m$ is actually transmitted serves as useful side information for the destination detection. With orthogonal signalings such as $M$-FSK, the transition from the correct message $m$ to any one of the $M-1$ incorrect message is equiprobable [31]. Thus, the transition probability for $M$-FSK can be expressed in terms of the SER, $\varepsilon_{r}^{\mathrm{f}}$, as follows:

$$
\operatorname{Pr}\left(m_{r} \mid m\right)=\left\{\begin{array}{cc}
1-\varepsilon_{r}^{\mathrm{f}}, & m_{r}=m \\
\frac{\varepsilon_{r}^{\mathrm{f}}}{M-1}, & m_{r} \neq m
\end{array}\right.
$$

where

$$
\varepsilon_{r}^{\mathrm{f}} \triangleq \sum_{m=1}^{M-1}(-1)^{m+1}\left(\begin{array}{c}
M-1 \\
m
\end{array}\right) \frac{1}{1+m\left(1+\gamma_{0 r}\right)} .
$$

For non-orthogonal signalings such as $M$-DPSK, however, the transition errors are not equiprobable because each constellation point is not equidistant from all other constellation points [31]. Thus, the simple relationship in (29) for $M$-FSK does not hold for $M$-DPSK. With non-trivial derivations, we derive the exact transition probability for $M$-DPSK as follows.

Lemma 5 (Exact Transition Probability for M-DPSK): For the MLD of M-DPSK in 27) at relay $\mathrm{T}_{r}, r=1, \cdots, K$, the exact transition probability in Rayleigh fading is given by $\operatorname{Pr}\left(m_{r} \mid m\right)=\mathcal{P}_{\left|m_{r}-m\right|}\left(\gamma_{0 r}\right)$, where

$\mathcal{P}_{n}\left(\gamma_{0 r}\right)=\frac{1}{2 \pi} \int_{\frac{2 n-1}{M} \pi}^{\frac{2 n+1}{M} \pi} \frac{1}{1+\frac{\gamma_{0 r}^{2} \sin ^{2} \theta}{1+2 \gamma_{0 r}}}\left[1+\frac{\gamma_{0 r} \cos \theta}{\sqrt{\gamma_{0 r}^{2} \sin ^{2} \theta+2 \gamma_{0 r}+1}} \arccos \left(-\frac{\gamma_{0 r} \cos \theta}{1+\gamma_{0 r}}\right)\right] \mathrm{d} \theta$,

$n=0,1, \cdots, M-1$.

Proof: See Appendix D.

The exact transition probability of $M$-DPSK depends only on the transition phase indexed by $\left|m_{r}-m\right|$. It is not hard to mathematically prove $\mathcal{P}_{n}\left(\gamma_{0 r}\right)=\mathcal{P}_{M-n}\left(\gamma_{0 r}\right)$ for $n=0, \cdots, M-1$. This property can be attributed to the symmetry of the PSK constellations. It suggests that in order to characterize $M$ transition probabilities for $M \geq 4$, one only needs to calculate $M / 2$ transition probabilities $\mathcal{P}_{n}\left(\gamma_{0 r}\right)$ for $n=0, \cdots, \frac{M}{2}-1$ via (31), and the remaining $M / 2$ 
transition probabilities, $\mathcal{P}_{n}\left(\gamma_{0 r}\right), n=\frac{M}{2}, \cdots, M$, follow immediately by the symmetry $\mathcal{P}_{n}\left(\gamma_{0 r}\right)=$ $\mathcal{P}_{M-n}\left(\gamma_{0 r}\right)$.

Lemma 6 (Closed-Form Approx. Transition Probability): The transition probability of M-DPSK in Rayleigh fading can be approximated as

$$
\operatorname{Pr}\left(m_{r} \mid m\right) \approx \begin{cases}1-\varepsilon^{\mathrm{p}}, & m_{r}=m, \\ \frac{\varepsilon^{\mathrm{p}}}{2}, & \left|m_{r}-m\right|=1 \text { or } M-1, \\ 0, & \text { otherwise }\end{cases}
$$

where

$$
\varepsilon_{r}^{\mathrm{p} \triangleq} \begin{cases}\frac{1}{2\left(1+\gamma_{0 r}\right)}, & M=2, \\ 1.03 \sqrt{\frac{1+\cos \frac{\pi}{M}}{2 \cos \frac{\pi}{M}}}\left[1-\sqrt{\frac{\left(1-\cos \frac{\pi}{M}\right) \gamma_{0 r}}{1+\left(1-\cos \frac{\pi}{M}\right) \gamma_{0 r}}}\right], & M \geq 4 .\end{cases}
$$

Proof: See Appendix E.

The approximate transition probability in (32) exploits the fact that the error transmissions from the transmitted PSK phase to its two closest neighbouring phases are most likely and euqi-probable. Moreover, error transitions to any farther constellations points other than the two closest constellation points are negligible.

\section{Unified MLDs at the Destination}

In this subsection, we develop the MLDs at the destination for both PS-NcDF and TS-NcDF protocols in a unified form, which exploits explicitly the transition probabilities at the relays to ameliorate the error propagations from the relays.

Theorem 1 (Exact MLDs): The exact MLDs of $M$-DPSK at the destination for both PS-NcDF and TS-NcDF are given in a unified form as follows:

$\hat{m}=\underset{m=0, \cdots, M-1}{\arg \max }\left\{\frac{2 \gamma_{0 d}}{1+2 \gamma_{0 d}} \beta_{0}(m)+\sum_{r=1}^{K} \ln \left[\sum_{m_{r}=0}^{M-1} \mathcal{P}_{\left|m_{r}-m\right|}\left(\gamma_{0 r}\right) \mathrm{e}^{-\beta_{r}^{-}\left(m_{r}\right)} I\left(2 \gamma_{r d}, \beta_{r}^{+}\left(m_{r}\right)\right)\right]\right\}$,

where $\beta_{0}(m) \triangleq \frac{\Re\left\{y_{0 d}(l-1) y_{0 d}^{*}(l) \mathrm{e}^{j 2 \pi m / M}\right\}}{\sigma_{0 d}^{2}}, \beta_{r}^{+}\left(m_{r}\right) \triangleq \frac{\left|y_{r d}(l)+y_{r d}(l-1) \mathrm{e}^{j 2 \pi m_{r} / M}\right|^{2}}{2 \sigma_{r d}^{2}}, \beta_{r}^{-}\left(m_{r}\right) \triangleq \frac{\left|y_{r d}(l)-y_{r d}(l-1) \mathrm{e}^{j 2 \pi m_{r} / M}\right|^{2}}{2 \sigma_{r d}^{2}}$, and $\mathcal{P}_{\left|m_{r}-m\right|}\left(\gamma_{0 r}\right)$ is the transition probability given in (31). Similarly, the exact MLDs of $M$-FSK at the destination for PS-NcDF and TS-NcDF are given in a unified form as

$$
\hat{m}=\arg \max _{m=0, \cdots, M-1}\left\{\frac{\gamma_{0 d}}{1+\gamma_{0 d}} \frac{\left|\mathrm{y}_{0 d}(m+1)\right|^{2}}{\sigma_{0 d}^{2}}+\sum_{r=1}^{K} \ln \left[\left(1-\varepsilon_{r}^{\mathrm{f}}\right) \mathrm{e}^{-\frac{\left\|\mathbf{y}_{r d}\right\|^{2}-\left.\right|_{\left.\mathrm{y}_{r d}(m+1)\right|^{2}}}{\sigma_{r d}^{2}}}\right.\right.
$$




$$
\left.\left.\times I\left(\gamma_{r d}, \frac{\left|\mathrm{y}_{r d}(m+1)\right|^{2}}{\sigma_{r d}^{2}}\right)+\frac{\varepsilon_{r}^{\mathrm{f}}}{M-1} \sum_{\substack{m_{r}=0 \\ m_{r} \neq m}}^{M-1} \mathrm{e}^{-\frac{\left\|\mathrm{y}_{r d}\right\|^{2}-\left|\mathrm{y}_{r d}\left(m_{r}+1\right)\right|^{2}}{\sigma_{r d}^{2}}} I\left(\gamma_{r d}, \frac{\left|\mathrm{y}_{r d}\left(m_{r}+1\right)\right|^{2}}{\sigma_{r d}^{2}}\right)\right]\right\}
$$

Proof: See Appendix F.

The exact (optimum) MLDs in (34) and (35) require the evaluations of the integral $I(\epsilon, \beta)$ in (20). In addition, the transition probability involved in the exact MLD of (34) is given by a finite integral-form in (31), which makes the exact MLDs complex to implement in practice. Nevertheless, it is very meaningful to study the exact MLDs as they serve as the optimum performance benchmark for SWIPT in noncoherent DF systems. With the exact MLDs, one may use them as a theoretical performance upper bound to evaluate the performance of suboptimum detectors, if developed. In the following, to make the MLDs suitable for practical systems, we develop closed-form approximations for the MLDs.

Theorem 2 (Approximate MLDs): For $M$-DPSK, the unified-form approximate MLDs for both PS-NcDF and TS-NcDF are given by

$$
\hat{m} \approx \begin{cases}\arg \max _{m=0, \cdots, M-1}\left\{\frac{2 \gamma_{0 d}}{1+2 \gamma_{0 d}} \beta_{0}(m)+\sum_{r=1}^{K} \ln \left[\left(1-\varepsilon_{r}^{\mathrm{p}}\right) \mathrm{e}^{-\beta_{r}^{-}(m)} I_{2}\left(2 \gamma_{r d}, \beta_{r}^{+}(m)\right)\right.\right. & \\ \left.\left.+\varepsilon_{r}^{\mathrm{p}} \mathrm{e}^{-\beta_{r}^{-}(m+1)} I_{2}\left(2 \gamma_{r d}, \beta_{r}^{+}(m+1)\right)\right]\right\}, & M=2, \\ \arg \max _{m=0, \cdots, M-1}\left\{\frac{2 \gamma_{0 d}}{1+2 \gamma_{0 d}} \beta_{0}(m)+\sum_{r=1}^{K} \ln \left[\left(1-\varepsilon_{r}^{\mathrm{p}}\right) \mathrm{e}^{-\beta_{r}^{-}(m)} I_{2}\left(2 \gamma_{r d}, \beta_{r}^{+}(m)\right)\right.\right. & \\ \left.\left.+\varepsilon_{r}^{\mathrm{p}} \frac{\mathrm{e}^{-\beta_{r}^{-}(m+1)} I_{2}\left(2 \gamma_{r d}, \beta_{r}^{+}(m+1)\right)+\mathrm{e}^{-\beta_{r}^{-}(m-1)} I_{2}\left(2 \gamma_{r d}, \beta_{r}^{+}(m-1)\right)}{2}\right]\right\}, & M \geq 4 .\end{cases}
$$

For $M$-FSK, the approximate MLDs for both PS-NcDF and TS-NcDF are unified as

$$
\begin{gathered}
\hat{m} \approx \arg \max _{m=0, \cdots, M-1}\left\{\frac{\gamma_{0 d}}{1+\gamma_{0 d}} \frac{\left|\mathrm{y}_{0 d}(m+1)\right|^{2}}{\sigma_{0 d}^{2}}+\sum_{r=1}^{K} \ln \left[\left(1-\varepsilon_{r}^{\mathrm{f}}\right) \mathrm{e}^{-\frac{\left\|\mathbf{y}_{r d}\right\|^{2}-\left|\mathrm{y}_{r d}(m+1)\right|^{2}}{\sigma_{r d}^{2}}}\right.\right. \\
\left.\left.\times I_{2}\left(\gamma_{r d}, \frac{\left|\mathrm{y}_{r d}(m+1)\right|^{2}}{\sigma_{r d}^{2}}\right)+\frac{\varepsilon_{r}^{\mathrm{f}}}{M-1} \sum_{\substack{m_{r}=0 \\
m_{r} \neq m}}^{M-1} \mathrm{e}^{-\frac{\left\|\mathbf{y}_{r d}\right\|^{2}-\left|\mathrm{y}_{r d}\left(m_{r}+1\right)\right|^{2}}{\sigma_{r d}^{2}}} I_{2}\left(\gamma_{r d}, \frac{\left|\mathrm{y}_{r d}\left(m_{r}+1\right)\right|^{2}}{\sigma_{r d}^{2}}\right)\right]\right\} .
\end{gathered}
$$

Proof: Following the exact MLDs in Theorem 1 applying the approximation of $I(\epsilon, \beta) \approx$ $I_{2}(\epsilon, \beta)$ for $M$-FSK and $M$-DPSK and the transition probability approximation in Lemma 6 for $M$-DPSK immediately gives the approximate MLDs.

The ML decision metrics for the exact MLDs in Theorem 1 are given by the weighted sum of the decision statistics associated with the direct link and the relay branches. For each relay branch, the weight function is proportional to the transition probability and the decision statistic 
is involved in the integral function $I(\epsilon, \beta)$. For the approximate MLDs in Theorem 2, the exact integral function $I(\epsilon, \beta)$ is replaced by its tight closed-form approximation $I_{2}(\epsilon, \beta)$. In addition, for $M$-DPSK, the approximate transition probability is used in place of the exact transition probability, which reduces the sum of $M-1$ transition terms to 2 terms, irrespective of $M$.

\section{NUMERICAL RESUltS}

In this section, we carry out Monte Carlo simulations to gain useful design insights into the proposed PS-NcDF and TS-NcDF protocols. The EH efficiency at the relays is set to $\eta=0.6$ as in [2]. We adopt the bounded path-loss model as in [21] in which the path-loss is strictly larger than one. Specifically, the path-loss from transmit terminal $i$ to receive terminal $j$ is modeled by $\mathcal{L}_{i j}=\frac{1}{1+D_{i j}^{\varrho}}$, where $\varrho$ is the path-loss exponent and $D_{i j}$ is the transmitter-receiver distance for $i j \in\{0 d, 0 r, r d\}_{r=1}^{K}$. The path-loss exponent is set to $\varrho=2.7$ which corresponds to the urban cellular communication environment [32]. The source-destination distance is fixed to $D_{0 d}=3$ (m), while the source-relay distance $D_{0 r}$ and relay-destination distance $D_{r d}$ can vary arbitrarily between 0 and 3 subject to $D_{0 r}+D_{r d}=3$, for $r=1, \cdots, K$. For ease of simulations, the noise variances are set to be $\sigma_{i j, 1}^{2}=\sigma_{i j, 2}^{2} \triangleq \sigma_{0}^{2} / 2$, such that each receive terminal has a total noise variance $\sigma_{0}^{2}$, i.e., $\sigma_{i j, 1}^{2}+\sigma_{i j, 2}^{2}=\sigma_{0}^{2}$, where $\sigma_{i j, 1}^{2}$ and $\sigma_{i j, 2}^{2}, i j \in\{0 d, 0 r, r d\}_{r=1}^{K}$, are the variances of the receive antenna noise and the ID circuit noise, respectively. The error performance is parameterized by the transmitter $S N R$, defined as $\mathrm{SNR} \triangleq P_{0} / \sigma_{0}^{2}$.

We will evaluate the error performance of PS-NcDF and TS-NcDF at the same information rate $R \triangleq \frac{N_{s} \log _{2} M}{T}$ (bps), where $N_{s}$ is the total number of information-bearing symbols transmitted over $T$ (sec). Note that $N_{s}$ new symbols are transmitted in the first block, which are relayed in the following $K$ blocks by the relays. The symbol time $T_{s}$ is related to $R$ as

$$
T_{s}= \begin{cases}\frac{\log _{2} M}{(K+1) R}, & \text { PS-NcDF } \\ \frac{(1-\alpha) \log _{2} M}{(K+1) R}, & \text { TS-NcDF }\end{cases}
$$

For simplicity, we will fix $N_{s} / T=1$ such that $R=\log _{2} M$, which depends only on $M$.

\section{A. Validation of the Piecewise Approximation $I_{2}(\epsilon, \beta)$ in (26)}

We first check the accuracy of the proposed piecewise approximation $I_{2}(\epsilon, \beta)$ in (26). As a benchmark, we use the MATLAB numerical integration function "integral" to directly compute 


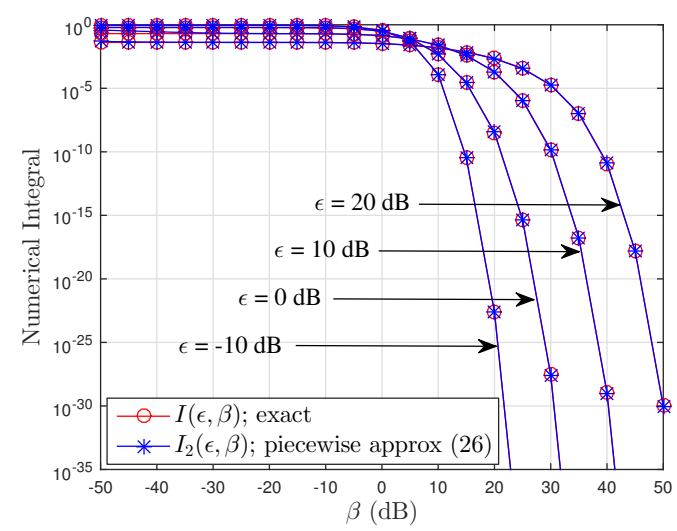

Fig. 2. Piecewise closed-form approximation $I_{2}(\epsilon, \beta)$ vs the exact numerical integration $I(\epsilon, \beta)$ for various $\epsilon$ and $\beta$ values.

$I(\epsilon, \beta)$ in (20) at a relative error tolerance of $10^{-12}$, which is referred to as the "exact" result. We evaluate $I_{2}(\epsilon, \beta)$ for $\beta \in[-50,50] \mathrm{dB}$ under various $\epsilon$ values of interest ranging from -10 to $20 \mathrm{~dB}$ in Fig. 2. Since $\beta$ spans over a very wide range of values for each $\epsilon$, the three subcases involved in (26) are all considered in Fig. 2 for each $\epsilon$ value. In this case, the piecewise approximation of (26) is applied according to the conditions for the subcases. It can be observed that $I_{2}(\epsilon, \beta)$ and $I(\epsilon, \beta)$ overlap for all possible values of $\epsilon$ and $\beta$, which confirms that $I_{2}(\epsilon, \beta)$ in (26) is indeed a very good approximation.

\section{B. Validation of the Approximate MLDs in (36) and (37)}

We validate the proposed closed-form approximate MLDs in (36) and (37) through comparisons to the exact (integral-form) MLDs in (34) and (35), for binary DPSK (BDPSK) and binary FSK (BFSK) signalings with fixed symbol rate $R=1$ (bps). Specifically, the TS-NcDF protocol with time switching coefficient $\alpha=0.5$ in a single-relay $(K=1) \mathrm{EH}$ system with $D_{0 r}=1.5$ (m) is considered in Fig. 3(a), and the PS-NcDF protocol with power splitting factor $\rho=0.5$ for a two-relay $(K=2) \mathrm{EH}$ system with $D_{01}=1(\mathrm{~m})$ and $D_{02}=2(\mathrm{~m})$ is considered in Fig. 3(b) As demonstrated in these figures, the SERs of the approximate MLDs are in excellent agreement with those of the exact MLDs over the whole SNR range, indicating that the proposed closed-form approximate MLDs are useful practical solutions for noncoherent SWIPT.

\section{Comparison of PS-NcDF and TS-NcDF}

We now compare PS-NcDF and TS-NcDF in terms of the SER at the same information rate $R$. Fig. 4 shows the SER versus the time switching coefficient $\alpha$ for TS-NcDF or power splitting 


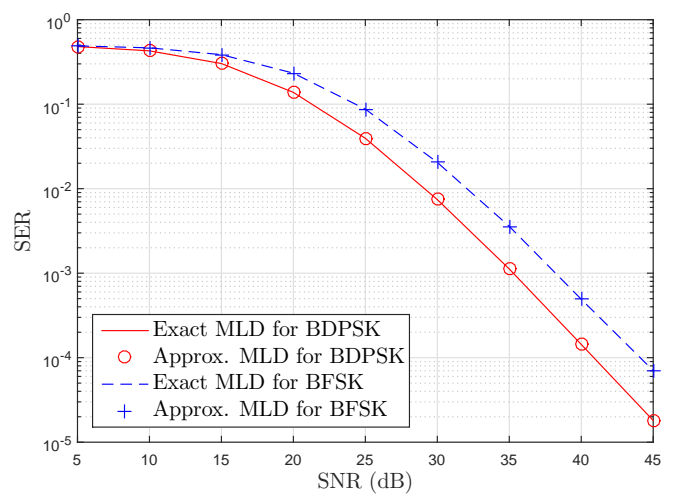

(a)

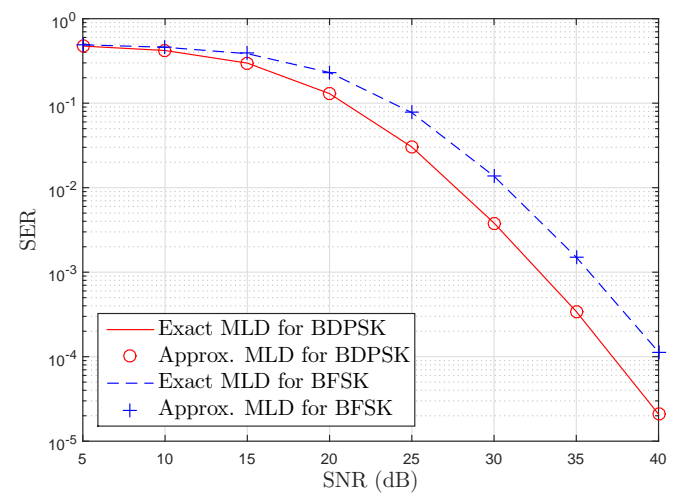

(b)

Fig. 3. SERs of MLDs for binary signalings $(M=2)$ with $R=1$ (bps). (a) TS-NcDF with $\alpha=0.5$ in a single-relay network $(K=1)$ with $D_{0 r}=1.5(\mathrm{~m})$. (b) PS-NcDF with $\rho=0.5$ in a two-relay network $(K=2)$ with $D_{01}=1(\mathrm{~m})$ and $D_{02}=2(\mathrm{~m})$

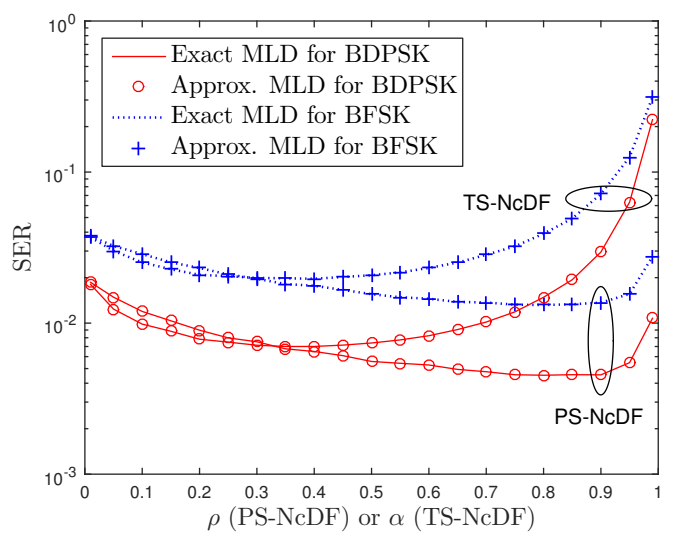

(a)

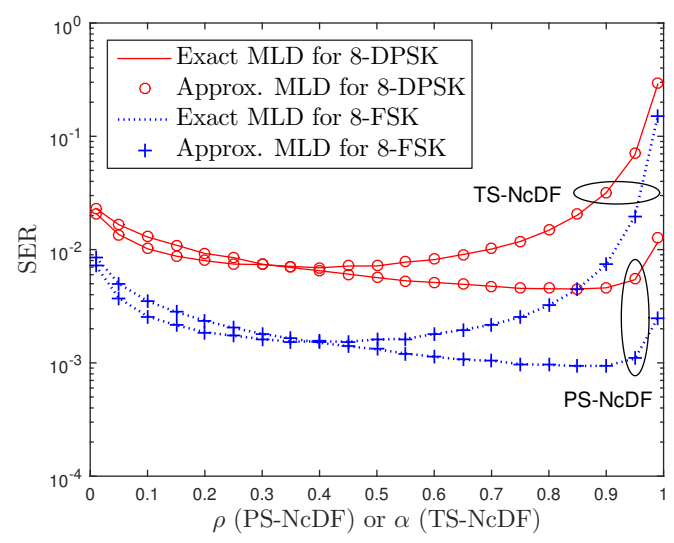

(b)

Fig. 4. Comparison of PS-NcDF and TS-NcDF for fixed information rate in a single-relay network $(K=1)$ with $D_{0 r}=1.5$ (m): (a) $M=2, \mathrm{SNR}=30 \mathrm{~dB}$, and $R=1$ (bps); and (b) $M=8, \mathrm{SNR}=40 \mathrm{~dB}$, and $R=3$ (bps).

factor $\rho$ for PS-NcDF in a single-relay $(K=1)$ network with $D_{0 r}=1.5(\mathrm{~m})$, where binary signalings $(M=2)$ with $R=1$ (bps) and $\mathrm{SNR}=30 \mathrm{~dB}$ (see Fig. 4(a)) and octonary signalings ( $M=8$ ) with $R=3$ (bps) and $\mathrm{SNR}=40 \mathrm{~dB}$ (see Fig. 4(b)) are considered. As shown in Fig. 4, the SERs exhibit some global minima around $\alpha=0.4$ for TS-NcDF and around $\rho=0.8$ for PS-NcDF. This is because there exists a performance tradeoff between the first and second hops through the adjustment of $\alpha$ (for TS-NcDF) or $\rho$ (for PS-NcDF). Specifically, when $\alpha$ (or $\rho$ ) increases, more power (proportional to $\alpha$ or $\rho$ ) can be harvested and utilized by the second-hop data relaying, resulting in an increased SNR over the second-hop. On the other hand, for TS-NcDF, as $\alpha$ increases, less time (proportional to $1-\alpha$ ) is available for the first-hop information transmission. Thus, the symbol time $T_{s}$ for TS-NcDF must decrease so as 


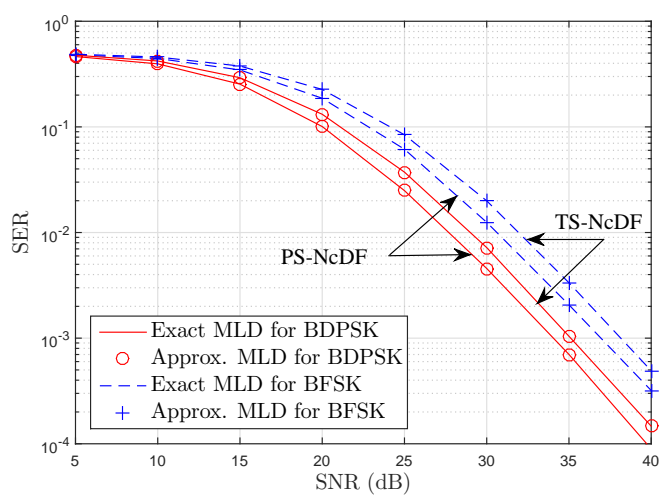

(a) $M=2$

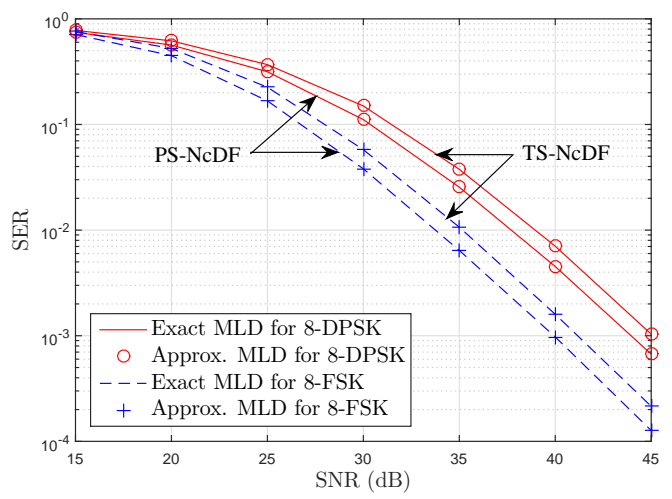

(c) $M=8$

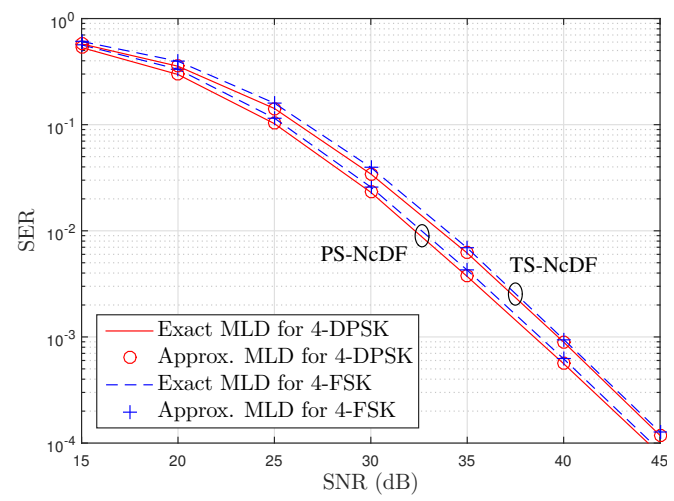

(b) $M=4$

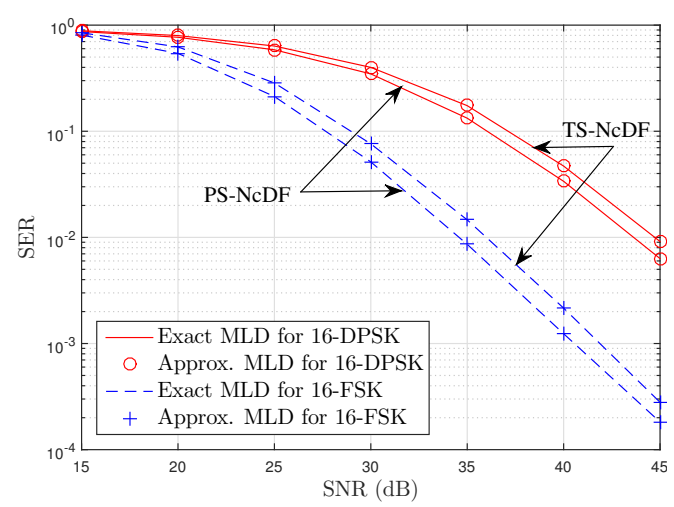

(d) $M=16$

Fig. 5. SERs of PS-NcDF and TS-NcDF employing near-optimal EH parameters with $\rho=0.8$ and $\alpha=0.4$ for a single relay network $(K=1)$ with $D_{0 r}=1.5$ (m): (a) $M=2$; (b) $M=4$; (c) $M=8$; and (d) $M=16$.

to maintain the same target information rate $R$, which results in smaller received symbol energy and consequently decreased SNR over the first-hop. Similarly, for PS-NcDF, as $\rho$ increases, less power (proportional to $1-\rho$ ) is split into the WIT circuit for information decoding; thus, the first-hop SNR decreases. Therefore, adjusting the values of $\alpha$ (or $\rho$ ) results in different impacts on the SNRs over the two hops, and consequently, a tradeoff exists between the first- and secondhop SNRs through the adjustment of $\alpha$ (or $\rho$ ). This can be also explained mathematically. By substituting (38) into (11b), we see that $\gamma_{0 r}$ is monotonically decreasing in $\alpha$ (or $\rho$ ) and $\gamma_{r d}$ is monotonically increasing in $\alpha$ (or $\rho$ ). Due to the opposite behaviors of the first- and second-hop SNRs as a function of $\alpha$ (or $\rho$ ), the optimal value of $\alpha$ (or $\rho$ ) minimizing the overall error probability is strictly between 0 and 1 .

\section{Impact of the Modulation Alphabet Size $M$}


In this subsection, we are interested in evaluating the performance of a variety of noncoherent signalings for $M=2,4,8,16$. To that end, we focus on a single-relay network with $D_{0 r}=1.5$ (m) employing the near-optimum EH parameters, i.e., $\rho=0.8$ for PS-NcDF and $\alpha=0.4$ for TS-NcDF. Note that $\rho=0.8$ and $\alpha=0.4$ minimize, respectively, the SERs of PS-NcDF and TS-NcDF, as shown in Fig. 4. However, it is difficult to analytically determine the optimum EH parameters. An interesting phenomenon observed is that while $M$-DPSK outperforms $M$-FSK for $M \leq 4$, the performance gain diminishes as $M$ increases, as shown in Figs. 5(a) and 5(b), In particular, for $M=4$ shown in Fig. 5(b), the performance gain of DPSK over FSK is almost negligible. As $M$ increases to $M \geq 8$, we see that $M$-FSK achieves a significant gain over $M$-DPSK. In particular, for a target SER of $10^{-2}$, the performance gain of $M$-FSK over $M$ DPSK is approximately $4 \mathrm{~dB}$ and $8 \mathrm{~dB}$ for $M=8$ and 16, respectively, as shown in Figs. 5(c) and 5(d), This implies that $M$-FSK achieves a promising energy gain over $M$-DPSK for large $M$. However, it is important to note that this energy gain comes at the cost of higher required bandwidth. In particular, the minimum double-sideband (DSB) bandwidth at an intermediate frequency (IF) assuming ideal rectangular Nyquist filtering [33] is $W=1 / T_{s}$ for $M$-DPSK and $W=M / T_{s}$ for $M$-FSK ( $M$ times that of $M$-DPSK). Nevertheless, considering the significant energy gain, $M$-FSK may be a more suitable solution, especially when $M \geq 8$, for EH relay systems due to the limited energy.

\section{CONCLUSIONS}

We have proposed a noncoherent SWIPT framework embracing both PS-NcDF and TS$\mathrm{NcDF}$ in a unified form, supporting $M$-FSK and $M$-DPSK. Following this framework, we developed exact noncoherent MLDs for PS-NcDF and TS-NcDF in a unified form, which involves integral evaluations. Nevertheless, the exact MLDs are useful for characterizing the optimum performance benchmark for noncoherent SWIPT. Furthermore, to make the MLDs suitable for practical implementation, we developed closed-form approximate MLDs achieving near-optimum performance. Numerical results demonstrated a performance tradeoff between the first and second hops through the adjustment of the time switching coefficient or power splitting factors. Moreover, the optimal values of these system parameters corresponding to the minimum SER are strictly between 0 and 1 . Finally, we demonstrated that $M$-FSK is an energy-efficient solution for noncoherent SWIPT, especially when $M \geq 8$. 
The conditional distribution of $\boldsymbol{X}_{0}$ given $X_{2}=z$ is $\left.\boldsymbol{X}_{0}\right|_{X_{2}=z} \sim \mathcal{C N}\left(\mathbf{0}, \boldsymbol{\Sigma}_{0}\right)$, where

$$
\Sigma_{0}=\left[\begin{array}{cc}
\Omega_{1}|z|^{2}+\Omega_{0}, & \Omega_{1}|z|^{2} c^{*} \\
\Omega_{1}|z|^{2} c, & \Omega_{1}|z|^{2}|c|^{2}+\Omega_{0}
\end{array}\right]
$$

It can be shown that $\operatorname{det}\left(\boldsymbol{\Sigma}_{0}\right)=\Omega_{0} \Omega_{1}|z|^{2}\left(1+|c|^{2}\right)+\Omega_{0}^{2}$ and

$$
\Sigma_{0}^{-1}=\frac{1}{\operatorname{det}\left(\Sigma_{0}\right)}\left[\begin{array}{cc}
\Omega_{1}|z|^{2}|c|^{2}+\Omega_{0}, & -\Omega_{1}|z|^{2} c^{*} \\
-\Omega_{1}|z|^{2} c, & \Omega_{1}|z|^{2}+\Omega_{0}
\end{array}\right]
$$

After some manipulations, the conditional PDF is written as

$$
f_{\boldsymbol{X}_{0} \mid X_{2}=z}(\boldsymbol{x})=\frac{\exp \left(-\frac{\left|x_{2}-c x_{1}\right|^{2}}{\Omega_{0}\left(1+|c|^{2}\right)}\right)}{\pi^{2} \Omega_{0}^{2}} \frac{\exp \left(-\frac{\frac{\left|x_{1}+c^{*} x_{2}\right|^{2}}{\Omega_{0}\left(1+|c|^{2}\right)}}{1+\frac{\Omega_{1}}{\Omega_{0}}\left(1+|c|^{2}\right)|z|^{2}}\right)}{1+\frac{\Omega_{1}}{\Omega_{0}}\left(1+|c|^{2}\right)|z|^{2}} .
$$

The unconditional PDF is $f_{\boldsymbol{X}_{0}}(\boldsymbol{x})=\int_{x=0}^{\infty} f_{\boldsymbol{X}_{0}\left|X_{2}\right|^{2}=x}(\boldsymbol{x}) f_{\left|X_{2}\right|^{2}}(x) \mathrm{d} x$, which is evaluated to

$$
f_{\boldsymbol{X}_{0}}(\boldsymbol{x})=\frac{\mathrm{e}^{-\frac{\left|x_{2}-c x_{1}\right|^{2}}{\Omega_{0}\left(1+|c|^{2}\right)}}}{\pi^{2} \Omega_{0}^{2}} \int_{0}^{\infty} \frac{\mathrm{e}^{-\frac{\frac{\left|x_{1}+c^{*} x_{2}\right|^{2}}{\Omega_{0}\left(1+|c|^{2}\right)}}{1+\frac{\Omega_{1} \Omega_{2}}{\Omega_{0}\left(1+|c|^{2}\right) t}}}}{1+\frac{\Omega_{1} \Omega_{2}}{\Omega_{0}}\left(1+|c|^{2}\right) t} \mathrm{e}^{-t} \mathrm{~d} t
$$

Applying change of variable $x \leftarrow \frac{\frac{\left|x_{1}+c^{*} x_{2}\right|^{2}}{\Omega_{0}\left(1+|c|^{2}\right)}}{1+\frac{\Omega_{1} \Omega_{2}}{\Omega_{0}}\left(1+|c|^{2}\right) t}$ in $($ A.4 and after some manipulations, we have

$$
f_{\boldsymbol{X}_{0}}(\boldsymbol{x})=\frac{\mathrm{e}^{-\frac{\left|x_{2}-c x_{1}\right|^{2}}{\Omega_{0}\left(1+|c|^{2}\right)}}}{\pi^{2} \Omega_{0}^{2}} \frac{\mathrm{e}^{\frac{\Omega_{0}}{\Omega_{1} \Omega_{2}\left(1+|c|^{2}\right)}}}{\frac{\Omega_{1} \Omega_{2}}{\Omega_{0}}\left(1+|c|^{2}\right)} \int_{0}^{\frac{\left|x_{1}+c^{*} x_{2}\right|^{2}}{\Omega_{0}\left(1+|c|^{2}\right)}} \frac{1}{x} \mathrm{e}^{\left(x+\frac{\left|x_{1}+c^{*} x_{2}\right|}{\Omega_{1} \Omega_{2}\left(1+|c|^{2}\right)^{2} x}\right)} \mathrm{d} x
$$

which is the same as (18) upon some appropriate parameterizations.

The derivation of the PDF of $\boldsymbol{Y}_{0}$ follows similar lines as the derivation of $f_{\boldsymbol{X}_{0}}(\boldsymbol{x})$, which is briefly summarized as follows. The conditional PDF of $\boldsymbol{Y}_{0}$ given $\left|X_{2}\right|^{2}=x$ is given by

$$
f_{\left.\mathbf{Y}_{0}|| X_{2}\right|^{2}=x}(\boldsymbol{y})=\frac{1}{\left(\pi \Omega_{0}\right)^{M}} \exp \left(-\frac{1}{\Omega_{0}} \sum_{\substack{q=1 \\ q \neq p}}^{M}\left|y_{q}\right|^{2}\right) \frac{1}{1+\frac{\Omega_{1}}{\Omega_{0}} x} \exp \left(-\frac{\left|y_{p}\right|^{2}}{\Omega_{0}+\Omega_{1} x}\right) .
$$

Similarly, the unconditional PDF is obtained as

$$
f_{\boldsymbol{Y}_{0}}(\boldsymbol{y})=\frac{1}{\left(\pi \Omega_{0}\right)^{M}} \mathrm{e}^{-\frac{\|y\|^{2}-\left|y_{p}\right|^{2}}{\Omega_{0}}} \int_{0}^{\infty} \frac{1}{1+\frac{\Omega_{1}}{\Omega_{0}} x} \exp \left(-\frac{\left|y_{p}\right|^{2}}{\Omega_{0}+\Omega_{1} x}\right) \frac{1}{\Omega_{2}} \mathrm{e}^{-\frac{x}{\Omega_{2}}} \mathrm{~d} x .
$$

Using similar change of variable in (A.7), we can obtain the final expression of (19). 


\section{APPENDIX B: PRoOF OF LEMMA 2}

It follows that $\boldsymbol{Y}_{2} \sim \mathcal{C N}\left(\mathbf{0}, \boldsymbol{\Sigma}_{2}\right)$, where

$$
\Sigma_{2}=\Omega_{0}\left[\begin{array}{cc}
1+\gamma, & \gamma c^{*} \\
\gamma c, & 1+\gamma|c|^{2}
\end{array}\right]
$$

where $\gamma=\Omega_{1} / \Omega_{0}$. Applying the property of the multivariate Gaussian distribution, the conditional distribution of $Y_{2}$ given $Y_{1}$ follows a Gaussian distribution, i.e., $\left.Y_{2}\right|_{Y_{1}=y_{1}} \sim \mathcal{C N}\left(\vartheta_{0} y_{1}, \sigma_{0}^{2}\right)$, where $\vartheta_{0} \triangleq \frac{\gamma c}{1+\gamma}$ and $\sigma_{0}^{2} \triangleq \Omega_{0}\left(1+\frac{\gamma}{1+\gamma}|c|^{2}\right)$. Let $Z_{0} \triangleq Y_{1}^{*} Y_{2}$. It follows that $\left.Z_{0}\right|_{Y_{1}=y_{1}} \sim$ $\mathcal{C N}\left(\vartheta_{0}\left|y_{1}\right|^{2}, \sigma_{0}^{2}\left|y_{1}\right|^{2}\right)$. Thus, the unconditional PDF of $Z_{0}$ is

$$
f_{Z_{0}}(z)=\frac{\exp \left(\frac{2 \Re\left\{\vartheta_{0}^{*} z\right\}}{\sigma_{0}^{2}}\right)}{\pi \sigma_{0}^{2}\left(\Omega_{0}+\Omega_{1}\right)} \int_{0}^{\infty} \frac{1}{x} \exp \left\{-\left[\left(\frac{1}{\Omega_{0}+\Omega_{1}}+\frac{\left|\vartheta_{0}\right|^{2}}{\sigma_{0}^{2}}\right) x+\frac{|z|^{2}}{\sigma_{0}^{2} x}\right]\right\} \mathrm{d} x .
$$

According to [29, eq. (3.471.9)], (B.3) is evaluated to

$$
f_{Z_{0}}(z)=\frac{2}{\pi \sigma_{0}^{2}\left(\Omega_{0}+\Omega_{1}\right)} \exp \left(\frac{2 \Re\left\{\vartheta_{0}^{*} z\right\}}{\sigma_{0}^{2}}\right) K_{0}\left(2 \sqrt{\frac{1}{\Omega_{0}+\Omega_{1}}+\frac{\left|\vartheta_{0}\right|^{2}}{\sigma_{0}^{2}}} \frac{|z|}{\sigma_{0}}\right),
$$

where $K_{0}(x)$ is the zero-order modified Bessel function of the second kind [29, eq. (8.432.1)]. Using the cartesian-to-polar transformation, $R_{0} \mathrm{e}^{j \Theta_{0}} \triangleq Z_{0}$, the joint PDF of $R_{0}$ and $\Theta_{0}$ is

$$
f_{R_{0}, \Theta_{0}}\left(r_{0}, \theta_{0}\right)=\frac{2}{\pi \sigma_{0}^{2}\left(\Omega_{0}+\Omega_{1}\right)} r_{0} \exp \left[\frac{2\left|\vartheta_{0}\right| r_{0}}{\sigma_{0}^{2}} \cos \left(\theta_{0}-\measuredangle c\right)\right] K_{0}\left(\frac{2 r_{0}}{\sigma_{0}} \sqrt{\frac{1}{\Omega_{0}+\Omega_{1}}+\frac{\left|\vartheta_{0}\right|^{2}}{\sigma_{0}^{2}}}\right) \text {. }
$$

Since $\Theta=\Theta_{0}-\measuredangle c$, the PDF of $\Theta$ is $f_{\Theta}(\theta)=\int_{0}^{\infty} f_{R_{0}, \Theta_{0}}\left(r_{0}, \theta+\measuredangle c\right) \mathrm{d} r_{0}$, which evaluates to

$$
f_{\Theta}(\theta)=\frac{2}{\pi \sigma_{0}^{2}\left(\Omega_{0}+\Omega_{1}\right)} \int_{0}^{\infty} r_{0} \exp \left(\frac{2\left|\vartheta_{0}\right| r_{0}}{\sigma_{0}^{2}} \cos \theta\right) K_{0}\left(\frac{2 r_{0}}{\sigma_{0}} \sqrt{\frac{1}{\Omega_{0}+\Omega_{1}}+\frac{\left|\vartheta_{0}\right|^{2}}{\sigma_{0}^{2}}}\right) \mathrm{d} r_{0}
$$

It is verified by Mathematica ${ }^{\circledR}$ that the following equation holds 4

$$
\int_{0}^{\infty} x \mathrm{e}^{-a x} K_{0}(b x) d x=\frac{1}{b^{2}-a^{2}}-\frac{a \arccos \frac{a}{b}}{\left(b^{2}-a^{2}\right)^{3 / 2}}
$$

for $b>0$ and $\Re(a+b)>0$. Letting $a=-\frac{2\left|\vartheta_{0}\right| r_{0}}{\sigma_{0}^{2}} \cos \theta$ and $b=\frac{2}{\sigma_{0}} \sqrt{\frac{1}{\Omega_{0}+\Omega_{1}}+\frac{\left|\vartheta_{0}\right|^{2}}{\sigma_{0}^{2}}}$ in (B.6), we solve the integral of $(B .5)$ in closed-form as

$f_{\Theta}(\theta)=\frac{1}{2 \pi\left[1+\frac{\left|\vartheta_{0}\right|^{2}\left(\Omega_{0}+\Omega_{1}\right)}{\sigma_{0}^{2}} \sin ^{2} \theta\right]}\left[1+\frac{\cos \theta}{\sqrt{\sin ^{2} \theta+\frac{\sigma_{0}^{2}}{\left|\vartheta_{0}\right|^{2}\left(\Omega_{0}+\Omega_{1}\right)}}} \arccos \left(-\frac{\cos \theta}{\sqrt{\sin ^{2} \theta+\frac{\sigma_{0}^{2}}{\left|\vartheta_{0}\right|^{2}\left(\Omega_{0}+\Omega_{1}\right)}}}\right)\right]$.

Finally, substituting $\vartheta_{0}$ and $\sigma_{0}^{2}$ into the above expression yields the final expression in (21).

\footnotetext{
${ }^{4}$ An alternative closed-form solution of the integral in B.6 is available in [29, eq. (6.621.3)]. However, that expression is given in terms of the Gaussian hypergeometric function, which is more complex than the expression in (B.6).
} 


\section{APPENDIX C: PROOF OF LEMMA 4}

From (23), we can rewrite $I(\epsilon, \beta)$ for $\beta \epsilon>1$ as

$$
I(\epsilon, \beta)=\frac{\mathrm{e}^{\frac{1}{\epsilon}}}{\epsilon} K_{0}\left(2 \sqrt{\frac{\beta}{\epsilon}}\right)+\int_{\frac{1}{\epsilon}}^{\sqrt{\frac{\beta}{\epsilon}}} \frac{1}{\epsilon x} \mathrm{e}^{-\left(x+\frac{1}{\epsilon}\right)} \mathrm{e}^{-\frac{\beta}{\epsilon x}} \mathrm{~d} x .
$$

Over the integration interval $(1 / \epsilon, \sqrt{\beta / \epsilon})$ in (C.1), we have $0<\frac{1}{\epsilon x}<1,0<\mathrm{e}^{-\left(x+\frac{1}{\epsilon}\right)}<1$, and $0<\mathrm{e}^{-\frac{\beta}{\epsilon x}}<1$, meaning that the overall integrand is a finite bounded function. Moreover, the integrand is always between 0 and 1, which ensures fast convergence when approximated by the Gauss-Legendre quadrature (GLQ) [34]. Note that the GLQ is one of the most efficient and accurate non-adaptive quadrature rules for a given number of arithmetic operations [34]. Applying the $N$-th order GLQ rule of [30, eq. (25.4.29)] in (C.1) yields $I(\epsilon, \beta)=I_{N}(\epsilon, \beta)+R_{N}$, where $I_{N}(\epsilon, \beta)=\frac{\mathrm{e}^{\frac{1}{\epsilon}}}{\epsilon}\left[K_{0}\left(2 \sqrt{\frac{\beta}{\epsilon}}\right)+\Psi_{N}\left(\frac{1}{\epsilon}, \sqrt{\frac{\beta}{\epsilon}}\right)\right]$ and $\Psi_{N}(\cdot, \cdot)$ is given in (25). This gives the third case of (24). For $\beta \epsilon<1$ in (23), it is easy to verify that the overall integrand is not necessarily smaller than one in this case. Thus, simply applying the GLQ rule for $\beta \epsilon<1$ in (23) might give an approximation with slower convergence speed compared to the GLQ approximation for $\beta \epsilon>1$. However, if we further enforce $\frac{\mathrm{e}^{\frac{1}{\epsilon}}}{\epsilon} \leq 1$, then the pre-bracket coefficient $\frac{\mathrm{e}^{\frac{1}{\epsilon}}}{\epsilon}$ in (23) can scale down the approximation error for the integral, which can significantly accelerate the convergence speed for the approximation. Thus, for $\beta \epsilon<1$ and $\frac{\mathrm{e}^{\frac{1}{\epsilon}}}{\epsilon} \leq 1$, the $N$-th order GLQ rule of [30, eq. (25.4.29)] readily applies, which yields $I_{N}(\epsilon, \beta)=\frac{\mathrm{e}^{\frac{1}{\epsilon}}}{\epsilon}\left[K_{0}\left(2 \sqrt{\frac{\beta}{\epsilon}}\right)-\Psi_{N}\left(\beta, \sqrt{\frac{\beta}{\epsilon}}\right)\right]$. This is the same as the second case of (24). For the remaining case with $\beta \epsilon<1$ and $\frac{\mathrm{e}^{\frac{1}{\epsilon}}}{\epsilon}>1$, we start with the alternative form of $I(\epsilon, \beta)$ in (22), which can be written as $I(\epsilon, \beta)=\mathbb{E}\left[\frac{1}{1+\epsilon X_{0}} \exp \left(-\frac{\beta}{1+\epsilon X_{0}}\right)\right]$ where the expectation is taken with respect to $X_{0}$ and $X_{0}$ is an exponential random variable with unit mean. Thus, the integral $I(\epsilon, \beta)$ can be interpreted as the statistical mean of the random variable $g\left(X_{0}\right) \triangleq \frac{1}{1+\epsilon X_{0}} \exp \left(-\frac{\beta}{1+\epsilon X_{0}}\right)$. Using the $N$-th order estimate of the mean of random variables, we can approximate $I(\epsilon, \beta)$ by $I_{N}(\epsilon, \beta)$ as follows [26, eq. (5.85)]:

$$
I_{N}(\epsilon, \beta)=\left.\sum_{n=0}^{N} \frac{\mu_{n}}{n !} \frac{\mathrm{d}^{n} g(x)}{\mathrm{d} x}\right|_{x=E\left[X_{0}\right]=1}
$$

where $\mu_{n}=\mathbb{E}\left[\left|X_{0}-E\left[X_{0}\right]\right|^{n}\right], n=0, \cdots, N$. Using the Binomial theorem and the exponential distribution of $X_{0}$, it is not hard to show that $\mu_{n}=\sum_{i=0}^{n} \frac{(-1)^{i}}{i !} n$ !. The function $g(x)$ can be represented as a composite function with $g(x)=f_{0}\left[g_{0}(x)\right]$, where $f_{0}(t) \triangleq t \mathrm{e}^{-\beta t}$ and $t=g_{0}(x) \triangleq$ 
$1 /(1+\epsilon x)$. Thus, Faà di Bruno's formula [35] is applied as follows:

$$
\left.\frac{\mathrm{d}^{n} f_{0}\left[g_{0}(x)\right]}{\mathrm{d} x}\right|_{x=1}=\left.\left.\sum_{\substack{\sum_{i=1}^{n} i m_{i}=n \\ \forall m_{i} \in \mathbb{Z}^{+}}} \frac{n !}{\prod_{j=1}^{n} m_{j} !} f_{0}^{(\varsigma)}(t)\right|_{t=\tau} \prod_{k=1}^{n}\left(\frac{g_{0}^{(k)}(x)}{k !}\right)^{m_{k}}\right|_{x=1},
$$

where $\tau=g_{0}(1), \varsigma=\sum_{i=1}^{n} m_{i}$, and $\mathbb{Z}^{+} \triangleq\{0,1,2, \cdots\}$. It can be shown that $f_{0}^{(\varsigma)}(t)=$ $(\varsigma-\beta t)(-\beta)^{\varsigma-1} \mathrm{e}^{-\beta t}$ and $g_{0}^{(k)}(x)=\frac{(-1)^{k} k \epsilon^{k}}{(\epsilon x+1)^{k+1}}$. Applying these relationships in ([C.3), substituting the expression into $(\overline{C .2})$, and after tedious manipulations, we obtain the first case of (24).

\section{APPENDIX D: PROOF OF LEMMA 5}

According to the MLD rule in (27), the phase $\Theta_{r} \triangleq \measuredangle y_{0 r-1}^{*}(l) y_{0 r}(l)$ is a sufficient statistic for the MLD. Let $\mathcal{D}_{m} \triangleq\left\{\theta \mid \theta_{m}-\frac{\pi}{M} \leq \theta \leq \theta_{m}+\frac{\pi}{M}\right\}$ denote the decision region for the message $m$, where $\theta_{m} \triangleq \frac{2 \pi m}{M}, m=0, \cdots, M-1$. Then the ML decision rule is in favor of the message $m_{r}$ if $\Theta_{r} \in \mathcal{D}_{m_{r}}, m_{r}=0, \cdots, M-1$. Thus, the transition probability is written as

$$
\operatorname{Pr}\left(m_{r} \mid m\right)=\operatorname{Pr}\left(\frac{\left[2\left(m_{r}-m\right)-1\right] \pi}{M} \leq \Theta_{r}-\theta_{m} \leq \frac{\left[2\left(m_{r}-m\right)+1\right] \pi}{M} \mid m\right) .
$$

The unified system model for $\mathrm{T}_{r}$ in (13a) can be rewritten as $\boldsymbol{y}_{0 r}=\hbar_{0 r}\left[1, \mathrm{e}^{j \theta_{m}}\right]^{T}+\boldsymbol{n}_{0 r}^{\prime}$, where $\hbar_{0 r} \triangleq \sigma_{0 r} \sqrt{\gamma_{0 r}} h_{0 r} s(l-1)$ and $\boldsymbol{n}_{0 r}^{\prime} \triangleq \sigma_{0 r} \boldsymbol{n}_{0 r}$. It follows that $\hbar_{0 r} \sim \mathcal{C N}\left(0, \sigma_{0 r}^{2} \gamma_{0 r}\right)$ and $\boldsymbol{n}_{0 r}^{\prime} \sim \mathcal{C N}\left(0, \sigma_{0 r}^{2}\right)$. Applying the result in Lemma 2, the PDF of $\Theta_{r}^{\prime} \triangleq \Theta_{r}-\theta_{m}$ is given by

$$
f_{\Theta_{r}^{\prime}}(\theta)=\frac{1}{2 \pi} \frac{1}{1+\frac{\gamma_{0 r}^{2} \sin ^{2} \theta}{1+2 \gamma_{0 r}}}\left[1+\frac{\gamma_{0 r} \cos \theta}{\sqrt{\gamma_{0 r}^{2} \sin ^{2} \theta+2 \gamma_{0 r}+1}} \arccos \left(-\frac{\gamma_{0 r} \cos \theta}{\gamma_{0 r}+1}\right)\right] .
$$

Thus, the transition probability in (D.1) can be written as

$$
\begin{aligned}
\operatorname{Pr}\left(m_{r} \mid m\right) & =\int_{\frac{2\left(m_{r}-m\right)-1}{M} \pi}^{\frac{2\left(m_{r}-m\right)+1}{M} \pi} f_{\Theta_{r}^{\prime}}(\theta) \mathrm{d} \theta \\
& =\int_{\frac{2\left(m-m_{r}\right)-1}{M} \pi}^{\frac{2\left(m-m_{r}\right)+1}{M} \pi} f_{\Theta_{r}^{\prime}}(\alpha) \mathrm{d} \alpha \\
& =\operatorname{Pr}\left(m \mid m_{r}\right),
\end{aligned}
$$

where (D.3b) is due to the change of variable $\alpha \leftarrow-\theta$ and $f_{\Theta_{r}^{\prime}}(\theta)=f_{\Theta_{r}^{\prime}}(-\theta)$. Note that the symmetry $\operatorname{Pr}\left(m_{r} \mid m\right)=\operatorname{Pr}\left(m \mid m_{r}\right)$ can be also attributed to the rotational invariance property of the PSK signal constellation. According to $(\overline{D .3})$, the transition probability can be calculated as

$$
\operatorname{Pr}\left(m_{r} \mid m\right)=\int_{\frac{2\left|m_{r}-m\right|-1}{M} \pi}^{\frac{2\left|m_{r}-m\right|+1}{M} \pi} f_{\Theta_{r}^{\prime}}(\theta) \mathrm{d} \theta .
$$

Finally, substituting (D.2) into (D.4) yields the final expression in (31). 


\section{APPENDIX E: PROOF OF LEMMA 6}

Noticing that the transmitted PSK phase will most likely be mistaken for its two closest neighboring phases with equal probability [31], we can approximate the transition probability as follows: for the two closest error transitions with $\left|m_{r}-m\right|=1$ or $M-1$, we have $\operatorname{Pr}\left(m_{r} \mid m\right) \approx$ $\frac{\mathrm{SER}}{2}$; For the correct symbol transition with $m_{r}=m$, we have $\operatorname{Pr}\left(m_{r} \mid m\right)=1-\mathrm{SER}$. For all other error transitions, we simply ignore the probabilities. This approximation immediately gives (32). It remains to derive the SER for $M$-DPSK. For $M=2$, a closed-from exact expression of the SER is available, namely, $\mathrm{SER}=\varepsilon_{r}^{\mathrm{p}}=\frac{1}{2\left(1+\gamma_{0 r}\right)}$. For $M \geq 4$, however, no closed-form exact SER expression is reported in the literature for fading channels. To proceed, we resort to an upper bound on the SER for AWGN channels in [36, eq. (8.95)], which is the best known bound for AWGN channels. Taking the statistical average of this bound over the Rayleigh distribution by means of [29, eq. (6.283.2)], we obtain a novel upper bound on the (average) SER of $M$-DPSK as $\varepsilon_{r}^{\mathrm{p}}=1.03 \sqrt{\frac{1+\cos \frac{\pi}{M}}{2 \cos \frac{\pi}{M}}}\left[1-\sqrt{\frac{\left(1-\cos \frac{\pi}{M}\right) \gamma_{0 r}}{1+\left(1-\cos \frac{\pi}{M}\right) \gamma_{0 r}}}\right]$. Overall, the SER of $M$-DPSK is given by (33).

\section{APPENDIX F: PROOF OF THEOREM 1}

Applying Total Probability Theorem and Lemma 5, the likelihood function (LF) conditioned on message $m$ for the unified $M$-DPSK model in (13) can be written as

$$
f\left(\boldsymbol{y}_{0 d},\left\{\boldsymbol{y}_{r d}\right\}_{r=1}^{K} \mid m\right)=f\left(\boldsymbol{y}_{0 d} \mid m\right) \prod_{r=1}^{K} \sum_{m_{r}=0}^{M-1} f\left(\boldsymbol{y}_{r d} \mid m_{r}\right) \mathcal{P}_{\left|m_{r}-m\right|}\left(\gamma_{0 r}\right)
$$

From (13b), we see that $\left.\boldsymbol{y}_{0 d}\right|_{m} \sim \mathcal{C N}\left(\mathbf{0}, \boldsymbol{\Sigma}_{0 d}\right)$, where

$$
\boldsymbol{\Sigma}_{0 d}=\sigma_{0 d}^{2}\left[\begin{array}{cc}
1+\gamma_{0 d} & \gamma_{0 d} \mathrm{e}^{-j 2 \pi m / M} \\
\gamma_{0 d} \mathrm{e}^{j 2 \pi m / M} & 1+\gamma_{0 d}
\end{array}\right]
$$

After some manipulations, we have

$$
f\left(\boldsymbol{y}_{0 d} \mid m\right)=\frac{\exp \left(-\frac{\left(1+\gamma_{0 d}\right)\left\|\boldsymbol{y}_{0 d}\right\|^{2}}{\sigma_{0 d}^{2}\left(1+2 \gamma_{0 d}\right)}\right)}{\left(\pi \sigma_{0 d}^{2}\right)^{2}\left(1+2 \gamma_{0 d}\right)} \exp \left\{\frac{2 \gamma_{0 d}}{1+2 \gamma_{0 d}} \frac{\Re\left\{y_{0 d}(l-1) y_{0 d}^{*}(l) \mathrm{e}^{j 2 \pi m / M}\right\}}{\sigma_{0 d}^{2}}\right\} .
$$

From (13c), we see that applying Lemma 1 immediately yields the LF $f\left(\boldsymbol{y}_{r d} \mid m_{r}\right)$ as follows:

$$
f\left(\boldsymbol{y}_{r d} \mid m_{r}\right)=\frac{\exp \left(-\frac{\left|y_{r d}(l)-y_{r d}(l-1) \mathrm{e}^{j 2 \pi m_{r} / M}\right|^{2}}{2 \sigma_{r d}^{2}}\right)}{\left(\pi \sigma_{r d}^{2}\right)^{2}} I\left(2 \gamma_{r d}, \frac{\left|y_{r d}(l)+y_{r d}(l-1) \mathrm{e}^{j 2 \pi m_{r} / M}\right|^{2}}{2 \sigma_{r d}^{2}}\right) .
$$


Substituting ( $(\overrightarrow{F .3})$ and (F.4) into (F.1) and using the definitions of $\beta_{0}(m), \beta_{r}^{+}\left(m_{r}\right)$, and $\beta_{r}^{-}\left(m_{r}\right)$ given in Theorem 10 we obtain the MLD in (34) for $M$-DPSK. For $M$-FSK in (14), following similar procedure as in (F.1) for $M$-DPSK, the LF can be written as

$f\left(\mathbf{y}_{0 d},\left\{\mathbf{y}_{r d}\right\}_{r=1}^{K} \mid m\right)=f\left(\mathbf{y}_{0 d} \mid m\right) \prod_{r=1}^{K} \sum_{m_{r}=0}^{M-1}\left[\left(1-\epsilon_{r}\right) f\left(\mathbf{y}_{r d} \mid m_{r}=m\right)+\frac{\epsilon_{r}}{M-1} \sum_{m_{r} \neq m} f\left(\mathbf{y}_{r d} \mid m_{r}\right)\right]$,

Furthermore, the PDF of $\mathbf{y}_{0 d}$ in (14b), conditioned on the knowledge of $m$, is

$$
f\left(\mathbf{y}_{0 d} \mid m\right)=\frac{\mathrm{e}^{-\frac{\left\|\mathbf{y}_{0 d}\right\|^{2}}{\sigma_{0 d}^{2}}}}{\left(\pi \sigma_{0 d}^{2}\right)^{M}\left(1+\gamma_{0 d}\right)} \mathrm{e}^{\frac{\gamma_{0 d}}{1+\gamma_{0 d}} \frac{\mid \frac{\left|y_{0 d}(m+1)\right|^{2}}{\sigma_{0 d}^{2}}}{(n)}} .
$$

Applying Lemma 1 the PDF of $\mathbf{y}_{r d}$ in (14c) given $m_{r}$ is given by

$$
f\left(\mathbf{y}_{r d} \mid m_{r}\right)=\frac{1}{\left(\pi \sigma_{r d}^{2}\right)^{M}} \mathrm{e}^{-\frac{\left\|\mathbf{y}_{r d}\right\|^{2}-\left|\mathbf{y}_{r d}\left(m_{r}+1\right)\right|^{2}}{\sigma_{r d}^{2}}} I\left(\gamma_{r d}, \frac{\left|\mathrm{y}_{r d}\left(m_{r}+1\right)\right|^{2}}{\sigma_{r d}^{2}}\right) .
$$

Finally, substituting (F.6) and (F.7) into (F.5) yields the MLD in (35) for $M$-FSK.

\section{REFERENCES}

[1] X. Lu, P. Wang, D. Niyato, D. I. Kim, and Z. Han, "Wireless networks with RF energy harvesting: a contemporary survey," to appear in IEEE Commun. Surveys and Tutorials.

[2] X. Zhou, R. Zhang, and C. K. Ho, "Wireless information and power transfer: architecture design and rate-energy tradeoff," IEEE Trans. Commun., vol. 61, pp. 4754-4767, Nov. 2013.

[3] A. Goldsmith, Wireless Communications. Cambridge, U.K.: Cambridge Univ. Press, 2005.

[4] N. Shinohara, Wireless Power Transfer via Radiowaves. Hoboken, NJ: John Wiley \& Sons, Inc., 2014.

[5] L. R. Varshney, "Transporting information and energy simultaneously," in Proc. IEEE Int. Symp. Inf. Theory (ISIT), July 2008, pp. 1612-1616.

[6] P. Grover and A. Sahai, "Shannon meets Tesla: wireless information and power transfer," in Proc. IEEE Int. Symp. Inf. Theory (ISIT), June 2010, pp. 2363-2367.

[7] R. Zhang and C. K. Ho, "MIMO broadcasting for simultaneous wireless information and power transfer," IEEE Trans. Wireless Commun., vol. 12, pp. 1989-2001, May 2013.

[8] L. Liu, R. Zhang, and K.-C. Chua, "Wireless information transfer with opportunistic energy harvesting," IEEE Trans. Wireless Commun., vol. 12, pp. 288-300, Jan. 2013.

[9] —-, "Wireless information and power transfer: a dynamic power splitting approach," IEEE Trans. Commun., vol. 61, pp. 3990-4001, Sept. 2013.

[10] H. Ju and R. Zhang, "Throughput maximization in wireless powered communication networks," IEEE Trans. Wireless Commun., vol. 13, pp. 418-428, Jan. 2014.

[11] K. Huang and E. Larsson, "Simultaneous information and power transfer for broadband wireless systems," IEEE Trans. Signal Process., vol. 61, pp. 5972-5986, Dec. 2013.

[12] Z. Xiang and M. Tao, "Robust beamforming for wireless information and power transmission," IEEE Wireless Commun. Lett., vol. 1, pp. 372-375, Aug. 2012. 
[13] C. Xing, N. Wang, J. Ni, Z. Fei, and J. Kuang, "MIMO beamforming designs with partial CSI under energy harvesting constraints," IEEE Trans. Signal Process., vol. 20, pp. 363-366, Apr. 2013.

[14] H. Zhang, Y. Huang, S. Li, and L. Yang, "Energy-efficient precoder design for MIMO wiretap channels," IEEE Commun. Lett., vol. 18, pp. 1559-1562, Sept. 2014.

[15] A. A. Nasir, X. Zhou, S. Durrani, and R. A. Kennedy, "Relaying protocols for wireless energy harvesting and information processing," IEEE Trans. Wireless Commun., vol. 12, pp. 3622-3636, July 2013.

[16] B. Medepally and N. B. Mehta, "Voluntary energy harvesting relays and selection in cooperative wireless networks," IEEE Trans. Wireless Commun., vol. 9, pp. 3543-3553, Nov. 2010.

[17] I. Ahmed, A. Ikhlef, R. Schober, and R. K. Mallik, "Joint power allocation and relay selection in energy harvesting AF relay systems," IEEE Wireless Commun. Lett., vol. 2, pp. 239-242, Apr. 2013.

[18] I. Krikidis, S. Timotheou, and S. Sasaki, "RF energy transfer for cooperative networks: data relaying or energy harvesting?" IEEE Commun. Lett., vol. 16, pp. 1772-1775, Nov. 2012.

[19] Z. Ding and H. V. Poor, "Cooperative energy harvesting networks with spatially random users," IEEE Trans. Signal Process., vol. 20, pp. 1211-1214, Dec. 2013.

[20] Z. Ding, S. M. Perlaza, I. Esnaola, and H. V. Poor, "Power allocation strategies in energy harvesting wireless cooperative networks," IEEE Trans. Wireless Commun., vol. 13, pp. 846-860, Feb. 2014.

[21] Z. Ding, I. Krikidis, B. Sharif, and H. V. Poor, "Wireless information and power transfer in cooperative networks with spatially random relays," IEEE Trans. Wireless Commun., vol. 13, pp. 4440-4453, Aug. 2014.

[22] I. Krikidis, "Simultaneous information and energy transfer in large-scale networks with/without relaying," IEEE Trans. Commun., vol. 62, pp. 900-912, Mar. 2014.

[23] C. Xing, S. Ma, Z. Fei, Y.-C. Wu, and H. Poor, "A general robust linear transceiver design for multi-hop amplify-andforward MIMO relaying systems,” IEEE Trans. Signal Process., vol. 61, pp. 1196-1209, Mar. 2013.

[24] C. Xing, M. Xia, F. Gao, and Y.-C. Wu, "Robust transceiver with Tomlinson-Harashima precoding for amplify-and-forward MIMO relaying systems," IEEE J. Sel. Areas Commun., vol. 30, pp. 1370-1382, Sept. 2012.

[25] P. Liu, S. Gazor, I.-M. Kim, and D. I. Kim, "Noncoherent amplify-and-forward cooperative networks: robust detection and performance analysis," IEEE Trans. Commun., vol. 61, pp. 3644-3659, Sept. 2013.

[26] A. Papoulis and S. U. Pillai, Probability, Random Variables and Stochastic Processes, 4th ed. New York: McGraw-Hill, 2002.

[27] N. O’Donoughue and J. M. F. Moura, "On the product of independent complex Gaussians," IEEE Trans. Signal Process., vol. 60, pp. 1050-1063, Mar. 2012.

[28] R. F. Pawula, "Distribution of the phase angle between two vectors perturbed by Gaussian noise II," IEEE Trans. Veh. Technol., vol. 50, pp. 576-583, Mar. 2001.

[29] I. S. Gradshteyn and I. M. Ryzhik, Table of Integrals, Series, and Products, 7th ed. San Diego, CA: Academic Press, 2007.

[30] M. Abramowitz and I. A. Stegun, Handbook of Mathematical Functions with Formulas, Graphs, and Mathematical Tables. New York: Dover publications, 1964.

[31] J. G. Proakis, Digital Communications, 4th ed. New York: McGraw-Hill, 2000.

[32] T. S. Rappaport, Wireless Communications: Principle and Practice. Upper Saddle River, NJ: Prentice Hall, 2002.

[33] B. Sklar, Digital Communications: Fundamentals and Applications, 2nd ed. Upper Saddle River, NJ: Prentice Hall, 2001.

[34] P. J. Davis and P. Rabinowitz, Methods of Numerical Integration. Orlando, Florida: Academic Press, 1984. 
[35] W. P. Johnson, “The curious history of Faà di Bruno's formula,” American Mathematical Monthly, vol. 109, pp. 217-234, Mar. 2002.

[36] M. K. Simon and M.-S. Alouini, Digital Communications Over Fading Channels, 2nd ed. Hoboken, NJ: Wiley, 2005. 